\title{
Quadrature Rules and Iterative Method for Numerical Solution of Two-Dimensional Fuzzy Integral Equations
}

\author{
S. M. Sadatrasoul and R. Ezzati \\ Department of Mathematics, College of Basic Sciences, Karaj Branch, Islamic Azad University, Alborz, Iran \\ Correspondence should be addressed to R. Ezzati; ezati@kiau.ac.ir
}

Received 25 December 2013; Accepted 11 March 2014; Published 19 May 2014

Academic Editor: Soheil Salahshour

Copyright (c) 2014 S. M. Sadatrasoul and R. Ezzati. This is an open access article distributed under the Creative Commons Attribution License, which permits unrestricted use, distribution, and reproduction in any medium, provided the original work is properly cited.

\begin{abstract}
We introduce some generalized quadrature rules to approximate two-dimensional, Henstock integral of fuzzy-number-valued functions. We also give error bounds for mappings of bounded variation in terms of uniform modulus of continuity. Moreover, we propose an iterative procedure based on quadrature formula to solve two-dimensional linear fuzzy Fredholm integral equations of the second kind (2DFFLIE2), and we present the error estimation of the proposed method. Finally, some numerical experiments confirm the theoretical results and illustrate the accuracy of the method.
\end{abstract}

\section{Introduction}

The concept of fuzzy numbers and arithmetic operations with these numbers were first introduced and investigated by Zadeh and others. The topic of fuzzy integrations is discussed in [1]. The Henstock and Riemann integral for fuzzy-number-valued functions was introduced and studied in $[2,3]$. Their numerical computation was also proposed; see, for example, [3-6]. In [6], the authors obtained the upper estimates of error of some fuzzy quadrature rules for mappings of bounded variation and of Lipschitz type and gave some applications. In [7], the authors studied the Gaussian quadrature rules for fuzzy integrals. Also, in [8], Wu presented some optimal fuzzy quadrature formula for classes of fuzzy-number-valued functions of Lipschitz type. To study other works, see [9-12].

Since many real-valued problems in engineering and mechanics can be brought in the form of two-dimensional fuzzy integral equations, it is important that we develop quadrature rules and numerical methods for such integral equations. In this paper, we introduce two-dimensional fuzzy integrals and propose some generalized quadrature rules and their dependent theorems for mappings of bounded variation. Also, we present the conditions for existence of unique solution for 2DFFLIE2. Finally, we introduce an iterative method for solving 2DFFLIE2. The rest of the paper is organized as follows. In Section 2, we give basic information about the fuzzy set theory and develop them to two-dimensional space. Also, we define two-dimensional fuzzy integral equation and some other properties of it in this section. In Section 3, we derive the proposed method to obtain numerical solutions of 2DFFLIE2 based on an iterative procedure. The error estimation of the introduced method is presented in Section 4 in terms of uniform modulus of continuity to prove the convergence of the method. Some numerical experiments are presented in Section 5.

\section{Preliminaries}

In this section, we review some necessary basic definitions on fuzzy numbers, fuzzy-number-valued functions, and fuzzy integrals.

Definition 1 (see $[13,14])$. A fuzzy number is a function $u$ : $R \rightarrow[0,1]$ having the following properties:

(i) $u$ is normal; that is, $\exists x_{0} \in R$, such that $u\left(x_{0}\right)=1$;

(ii) $u$ is fuzzy convex set (i.e., $u(\lambda x+(1-\lambda) y) \geq$ $\min \{u(x), u(y)\}$, for all $x, y \in R, \lambda \in[0,1])$;

(iii) $u$ is upper semicontinuous on $R$; 
(iv) the support $\overline{\{x \in R: u(x)>0\}}$ is a compact set, where $\bar{A}$ denotes the closure of $A$.

The set of all fuzzy numbers is denoted by $R_{F}$. According to [2], any real number $\alpha \in R$ can be interpreted as a fuzzy number $\alpha=\chi_{\{\alpha\}}$, and therefore $R \subset R_{F}$. Also, the neutral element with respect to $\oplus$ in $R_{F}$ is denoted by $\widetilde{0}=\chi_{\{0\}}$.

Definition 2 (see $[2,15])$. For any $0<r \leq 1$, an arbitrary fuzzy number is represented in parametric form, by an ordered pair of functions $(\underline{u}(r), \bar{u}(r))$, which satisfies the following properties:

(i) $\underline{u}(r)$ is bounded left continuous nondecreasing function over $[0,1]$;

(ii) $\bar{u}(r)$ is bounded left continuous nonincreasing function over $[0,1]$;

(iii) $\underline{u}(r) \leq \bar{u}(r)$.

Moreover, the addition and scalar multiplication of fuzzy numbers in $R_{F}$ are defined as follows:

(i)

$$
(u \oplus v)(r)=(\underline{u}(r)+\underline{v}(r), \bar{u}(r)+\bar{v}(r)),
$$

(ii)

$$
(\lambda \odot v)(r)= \begin{cases}(\lambda \underline{u}(r), \lambda \bar{u}(r)) & \lambda \geq 0, \\ (\lambda \bar{u}(r), \lambda \underline{u}(r)) & \lambda<0 .\end{cases}
$$

Also, according to $[2,16]$, the following algebraic properties for any $u, v, w \in R_{F}$ hold:

(i) $u \oplus(v \oplus w)=(u \oplus v) \oplus w$;

(ii) $u \oplus \widetilde{0}=\widetilde{0} \oplus u=u$;

(iii) with respect to $\widetilde{0}$, none of $u \in\left(R_{F}-R\right), u \neq \widetilde{0}$ has opposite in $\left(R_{F},+\right)$;

(iv) $(a \oplus b) \odot u=a \odot u \oplus b \odot u$, for all $a, b \in R$ with $a b \geq 0$ or $a b \leq 0$;

(v) $a \odot(u \oplus v)=a \odot u \oplus a \odot v$, for all $a \in R$;

(vi) $a \odot(b \odot u)=(a b) \odot u$, for all $a \in R$ and $1 \odot u=u$.

Definition 3 (see $[2,17]$ ). For arbitrary fuzzy numbers $u=$ $(\underline{u}(r), \bar{u}(r)), \quad v=(\underline{v}(r), \bar{v}(r))$, the quantity $D(u, v)=$ $\sup _{r \in[0,1]} \max \{|\underline{u}(r)-\underline{v}(r)|,|\bar{u}(r)-\bar{v}(r)|\}$ is the distance between $u$ and $v$. Also, the following properties hold [6]:

(i) $\left(R_{F}, D\right)$ is a complete metric space;

(ii) $D(u \oplus w, v \oplus w)=D(u, v)$ for all $u, v, w \in R_{F}$;

(iii) $D(k \odot u, k \odot v)=|k| D(u, v)$ for all $u, v \in R_{F}$ for all $k \in$ $R$;

(iv) $D(u \oplus v, w \oplus e) \leq D(u, w)+D(v, e)$ for all $u, v, w, e \in R_{F}$;

(v) $D\left(k_{1} \odot u, k_{2} \odot u\right)=\left|k_{1}-k_{2}\right| D(u, \widetilde{0})$ for all $k_{1}, k_{2} \in R$ with $k_{1} k_{2} \geq 0$ and for all $u \in R_{F}$.

Throughout this paper, we denote that $\|\cdot\|_{F}=D(\cdot, 0)$.
Theorem 4 (see [14]). (i) $\left(R_{F}, D\right)$ is a complete metric space.

(ii) The pair $\left(R_{F}, D\right)$ is a commutative semigroup with $\widetilde{0}=$ $\chi_{0}$ zero elements but cannot be a group for pure fuzzy numbers.

(iii) $\|\cdot\|_{F}$ has the properties of a usual norm on $R_{F}$; that is, $\|\cdot\|_{F}=0$ if and only if $u=0,\|\lambda \odot u\|_{F}=|\lambda|\|u\|_{F}$, and $\|u \oplus v\|_{F} \leq\|u\|_{F}+\|v\|_{F}$.

(iv) $\left|\|u\|_{F}-\|v\|_{F}\right| \leq D(u, v)$ and $D(u, v) \leq\|u\|_{F}+\|v\|_{F}$ for any $u, v \in R_{F}$.

In [2], the authors introduced the concept of the Henstock integral for a fuzzy-number-valued function. We present a generalized definition of this concept for two-dimensional Henstock integrability for bivariate fuzzy-number-valued functions.

Definition 5. Suppose that $f:[a, b] \times[c, d] \rightarrow R_{F}$ is a bounded mapping, and then the function $\omega_{[a, b] \times[c, d]}(f, \cdot)$ : $R_{+} \cup 0 \rightarrow R_{+}$defined by

$$
\begin{array}{r}
\omega_{[a, b] \times[c, d]}(f, \delta)=\sup \{D(f(x, y), f(s, t)) ; \\
x, s \in[a, b] ; y, t \in[c, d] ; \\
\left.\sqrt{(x-s)^{2}+(y-t)^{2}} \leq \delta\right\}
\end{array}
$$

is called the modulus of oscillation of $f$ on $[a, b] \times[c, d]$.

Also, if $f \in C_{F}([a, b] \times[c, d])$ (i.e., $f:[a, b] \times[c, d] \rightarrow$ $R_{F}$ is continuous on $\left.[a, b] \times[c, d]\right)$, then $\omega_{[a, b] \times[c, d]}(f, \delta)$ is called uniform modulus of continuity of $f$. The following properties will be very useful in what follows. The proofs of these properties in one-dimensional case are presented in [14] and those in two-dimensional case will be obtained in a similar way.

Theorem 6. The following properties hold:

(i) $D(f(x, y), f(s, t))$ $\omega_{[a, b] \times[c, d]}\left(f, \sqrt{(x-s)^{2}+(y-t)^{2}}\right)$ for any $x, s \in[a, b]$ and $y, t \in[c, d]$

(ii) $\omega_{[a, b] \times[c, d]}(f, \delta)$ is a nondecreasing mapping in $\delta$;

(iii) $\omega_{[a, b] \times[c, d]}(f, 0)=0$;

(iv) $\omega_{[a, b] \times[c, d]}\left(f, \delta_{1}+\delta_{2}\right) \leq \omega_{[a, b] \times[c, d]}\left(f, \delta_{1}\right)+$ $\omega_{[a, b] \times[c, d]}\left(f, \delta_{2}\right)$ for any $\delta_{1}, \delta_{2} \geq 0 ;$

(v) $\omega_{[a, b] \times[c, d]}(f, n \delta) \leq n \omega_{[a, b] \times[c, d]}(f, \delta)$ for any $\delta \geq 0$ and $n \in N$;

(vi) $\omega_{[a, b] \times[c, d]}(f, \lambda \delta) \leq(\lambda+1) \omega_{[a, b] \times[c, d]}(f, \delta)$ for any $\delta, \lambda \geq 0$

Definition 7. Let $f:[a, b] \times[c, d] \rightarrow R_{F}$, for $\Delta_{x}^{n}: a=x_{0}<$ $x_{1}<\cdots<x_{n}=b$ and $\Delta_{y}^{n}: c=y_{0}<y_{1}<\cdots<y_{n}=d$, be two partitions of the intervals $[a, b]$ and $[c, d]$, respectively. Let one consider the intermediate points $\xi_{i} \in\left[x_{i-1}, x_{i}\right]$ and $\eta_{i} \in\left[y_{j-1}, y_{j}\right], i=1, \ldots, n ; j=1, \ldots, n$, and $\delta:[a, b] \rightarrow$ $R_{+}$and $\sigma:[c, d] \rightarrow R_{+}$. The divisions $P_{x}=\left(\left[x_{i-1}, x_{i}\right] ; \xi_{i}\right)$, $i=1, \ldots, n$, and $P_{y}=\left(\left[y_{j-1}, y_{j}\right] ; \eta_{j}\right), j=1, \ldots, n$, denoted shortly by $P_{x}=\left(\Delta^{n}, \xi\right)$ and $P_{y}=\left(\Delta^{n}, \eta\right)$ are said to be $\delta$-fine 
and $\sigma$-fine, respectively, if $\left[x_{i-1}, x_{i}\right] \subseteq\left(\xi_{i}-\delta\left(\xi_{i}\right), \xi_{i}+\delta\left(\xi_{i}\right)\right)$ and $\left[y_{j-1}, y_{j}\right] \subseteq\left(\eta_{j}-\sigma\left(\eta_{j}\right), \eta_{j}+\sigma\left(\eta_{j}\right)\right)$.

The function $f$ is said to be two-dimensional Henstock integrable to $I \in R_{F}$ if for every $\varepsilon>0$ there are functions $\delta:[a, b] \rightarrow R_{+}$and $\sigma:[c, d] \rightarrow R_{+}$such that for any $\delta$-fine and $\sigma$-fine divisions we have $D\left(\sum_{i=0}^{n} \sum_{j=0}^{n}\left(x_{i}-\right.\right.$ $\left.\left.x_{i-1}\right)\left(y_{j}-y_{j-1}\right) \odot f\left(\xi_{i}, \eta_{j}\right), I\right)<\epsilon$, where $\sum$ denotes the fuzzy summation. Then, $I$ is called the two-dimensional Henstock integral of $f$ and is denoted by $I(f)=(F H) \int_{c}^{d} \int_{a}^{b} f(s, t) d s d t$.

If the above $\delta$ and $\sigma$ are constant functions, then one recaptures the concept of Riemann integral. In this case, $I \in$ $R_{F}$ will be called two-dimensional integral of $f$ on $[a, b] \times$ $[c, d]$ and will be denoted by $(F R) \int_{c}^{d} \int_{a}^{b} f(s, t) d s d t$.

Corollary 8. In [13], the authors proved that if $f \in C_{F}[a, b]$, its definite integral exists, also (FR) $\int_{a}^{b} f(t ; r) d t=\int_{a}^{b} \underline{f}(t, r) d t$, and $\overline{(F R) \int_{a}^{b} f(t ; r) d t}=\int_{a}^{b} \bar{f}(t, r) d t$. In a similar way, we can prove that if $f \in C_{F}([a, b] \times[c, d])$, its definite integral exists, and one has

$$
\begin{aligned}
& \frac{(F R) \int_{c}^{d} \int_{a}^{b} f(s, t ; r) d s d t}{(F R) \int_{c}^{d} \int_{a}^{b} f(s, t ; r) d s d t}=\int_{c}^{d} \int_{a}^{b} \int_{a}^{b} \bar{f}(s, t, r) d s d t,
\end{aligned}
$$

Theorem 9. If $f$ and $g$ are Henstock integrable mappings on $[a, b] \times[c, d]$ and if $D(f(s, t), g(s, t))$ is Lebesgue integrable, then

$$
\begin{aligned}
& D\left((F H) \int_{c}^{d} \int_{a}^{b} f(s, t) d s d t,(F H) \int_{c}^{d} \int_{a}^{b} g(s, t) d s d t\right) \\
& \quad \leq(L) \int_{c}^{d} \int_{a}^{b} D(f(s, t), g(s, t)) d s d t .
\end{aligned}
$$

Proof. In [2, 17], the authors demonstrated that for any integrable functions $h, r:[\alpha, \beta] \rightarrow R_{F}$ we have $D\left((F H) \int_{\alpha}^{\beta} h(x) d x,(F H) \int_{\alpha}^{\beta} r(x) d x\right) \leq$ (L) $\int_{\alpha}^{\beta} D(h(x), r(x)) d x$, and, clearly, we obtain

$$
\begin{aligned}
& D\left((F H) \int_{c}^{d} \int_{a}^{b} f(s, t) d s d t,(F H) \int_{c}^{d} \int_{a}^{b} g(s, t) d s d t\right) \\
& \quad \leq(L) \int_{c}^{d} D\left((F H) \int_{a}^{b} f(s, t) d s,(F H) \int_{a}^{b} g(s, t) d s\right) d t \\
& \quad \leq(L) \int_{c}^{d} \int_{a}^{b} D(f(s, t), g(s, t)) d s d t
\end{aligned}
$$

which completes the proof.
Theorem 10. If $f:[a, b] \times[c, d] \rightarrow R_{F}$ is an integrable bounded mapping, then for any fixed $u \in[a, b]$ and $v \in[c, d]$ the function $\varphi_{u v}:[a, b] \times[c, d] \rightarrow R_{+}$, defined by $\varphi_{u v}(s, t)=$ $D(f(u, v), f(s, t))$, is Lebesgue integrable on $[a, b] \times[c, d]$.

Proof. Regarding [6], Lemma 1, part (ii), it is easy to see that if $f$ is two-dimensional Henstock integrable and bounded on $[a, b] \times[c, d]$, then $f_{-}^{r}(s, t)$ and $f_{+}^{r}(s, t)$ as real functions of $(s, t) \in[a, b] \times[c, d]$ are two-dimensional integrable and uniformly bounded with respect to $r \in[0,1]$; that is, $f_{-}^{r}(s, t)$ and $f_{+}^{r}(s, t)$ are Lebesgue measurable (as functions of $(s, t)$ ) and uniformly bounded with respect to $r \in[0,1]$ by

$$
\begin{aligned}
\varphi_{u v}(s, t)= & D(f(u, v), f(s, t)) \\
= & \sup _{r \in[0,1]} \max \left\{\left|f_{-}^{r}(u, v)-f_{-}^{r}(s, t)\right|,\right. \\
& \left.\left|f_{+}^{r}(u, v)-f_{+}^{r}(s, t)\right|\right\} \\
= & \sup _{r_{n} \in[0,1]} \max \left\{\left|f_{-}^{r_{n}}(u, v)-f_{-}^{r_{n}}(s, t)\right|,\right. \\
& \left.\left|f_{+}^{r_{n}}(u, v)-f_{+}^{r_{n}}(s, t)\right|\right\},
\end{aligned}
$$

where $r_{n}, n \in N$, represent all the rational numbers in $[0,1]$. By Lebesgue's theorem of dominated convergence, it follows that $\varphi_{u v}(s, t)$ is Lebesgue integrable on $[a, b] \times[c, d]$, and this ends the proof.

Definition 11. A function $f:[a, b] \times[c, d] \rightarrow R_{F}$ is said to be bounded if there exists $M$ such that $\|f(x, y)\|_{F} \leq M$ for any $(x, y) \in[a, b] \times[c, d]$.

Definition 12. A function $f:[a, b] \times[c, d] \rightarrow R_{F}$ is said to be of bounded variation if

$$
\sup _{(x, y) \in[a, b] \times[c, d]} V_{\Delta_{x y}^{n}}<\infty
$$

where

$$
V_{\Delta_{x y}^{n}}=\sum_{i=0}^{n-1} \sum_{j=0}^{n-1} D\left(f\left(x_{i+1}, y_{j+1}\right), f\left(x_{i}, y_{j}\right)\right)
$$

is the variation of $f$ related to partitions $\Delta_{x}^{n}, \Delta_{y}^{n}$. The total variation of $f$ is defined to be, in this case, the number

$$
\bigvee(f)=\sup _{(x, y) \in[a, b] \times[c, d]} V_{\Delta_{x y}^{n}} \in R
$$

It is known also that a function of bounded variation is Riemann integrable (see [18]), so it is Henstock integrable too.

Theorem 13. (i) If $[a, b] \times[c, d] \subseteq[e, f] \times[g, h]$, then $\omega_{[a, b] \times[c, d]}(f, \delta) \leq \omega_{[e, f] \times[g, h]}(f, \delta)$ for all $\delta \geq 0$.

(ii) If $f$ is of bounded variation, then $\omega_{[a, b] \times[c, d]}(f, \delta) \leq$ $\bigvee(f)$ for all $\delta \geq 0$. 
Proof. (i) It is easy to see that

$$
\begin{aligned}
& \sup \{D(f(x, y), f(s, t)) \mid x, s \in[a, b], y, t \in[c, d], \\
& \left.\sqrt{(x-s)^{2}+(y-t)^{2}} \leq \delta\right\} \\
& \leq \sup \{D(f(x, y), f(s, t)) \mid x, s \in[e, f], y, t \in[g, h], \\
& \left.\sqrt{(x-s)^{2}+(y-t)^{2}} \leq \delta\right\},
\end{aligned}
$$

and, therefore, we obtain the required inequality.

(ii) Let $x, s \in[a, b]$ and $y, t \in[c, d]$; assume that $a<$ $x<s<b, c<y<t<d, V_{\Delta x}=a=x_{0}<x_{1}=x<$ $x_{2}=s<b$, and $V_{\Delta y}=c=y_{0}<y_{1}=y<y_{2}=t<d$. Taking supremum for any $x, s \in[a, b]$ and $y, t \in[c, d]$ with $\sqrt{(x-s)^{2}+(y-t)^{2}} \leq \delta$, we obtain the required inequality. It is obvious now that under this condition $f$ is bounded; therefore, we obtain

$$
\begin{aligned}
\|f(x, y)\|_{F} & =D(f(x, y), \widetilde{0}) \\
& \leq D(f(x, y), f(a, c))+d(f(a, c), \widetilde{0}) \\
& \leq \bigvee(f)+\|f(a, c)\|_{F},
\end{aligned}
$$

which completes the proof.

Definition 14. A function $f:[a, b] \times[c, d] \rightarrow R_{F}$ is said to be L-Lipschitz, if

$$
D(f(x, y), f(s, t)) \leq L \sqrt{(x-s)^{2}+(y-t)^{2}},
$$

for any $x, s \in[a, b]$ and $y, t \in[c, d]$.

Definition 15. A function $f:[a, b] \times[c, d] \rightarrow R_{F}$ is said to be M-Condition, if

$$
D(f(x, y), f(s, t)) \leq M(b-a)(d-c),
$$

for any $x, s \in[a, b]$ and $y, t \in[c, d]$.

Remark 16. We see that if $f$ is $M$-Condition function, then $f$ is of bounded variation and

$$
\bigvee(f) \leq M(b-a)(d-c) .
$$

Indeed, we have

$$
\begin{aligned}
V_{\Delta_{x y}^{n}} & =\sum_{i=0}^{n-1} \sum_{j=0}^{n-1} D\left(f\left(x_{i+1}, y_{j+1}\right), f\left(x_{i}, y_{j}\right)\right) \\
& \leq \sum_{i=0}^{n-1} \sum_{j=0}^{n-1}\left(M\left(x_{i+1}-x_{i}\right)\left(y_{j+1}-y_{j}\right)\right) \\
& =M(b-a)(d-c),
\end{aligned}
$$

and since

$$
\bigvee(f)=\sup _{(x, y) \in[a, b] \times[c, d]} V_{\Delta_{x y}^{n}}
$$

we obtain the required result.

\section{Quadrature Rules for Two-Dimensional (2D) Henstock Integrals}

In this section, we present some quadrature rules for $2 \mathrm{D}$ Henstock integral. The following theorem gives a unified approach to quadrature rules in 2D Henstock integrals.

Theorem 17. Let $f:[c, d] \times[c, d] \rightarrow R_{F}$ be Henstock integrable, bounded mappings. Then, for any divisions $a=x_{0}<$ $x_{1}<\cdots<x_{n}=b$ and $c=y_{0}<y_{1}<\cdots<y_{n}=d$ and any points $\xi_{i} \in\left[x_{i-1}, x_{i}\right]$ and $\eta_{j} \in\left[y_{j-1}, y_{j}\right]$, one has

$$
\begin{aligned}
& D\left((F H) \int_{c}^{d}(F H) \int_{a}^{b} f(s, t) d s d t\right. \\
& \left.\quad \sum_{j=1}^{n} \sum_{i=1}^{n}\left(x_{i}-x_{i-1}\right)\left(y_{j}-y_{j-1}\right) \odot f\left(\xi_{i}, \eta_{j}\right)\right) \\
& \leq \sum_{j=1}^{n} \sum_{i=1}^{n}\left(x_{i}-x_{i-1}\right)\left(y_{j}-y_{j-1}\right) \omega_{\left[x_{i-1}, x_{i}\right] \times\left[y_{j-1}, y_{j}\right]} \\
& \cdot\left(f, \sqrt{\left(x_{i}-x_{i-1}\right)^{2}+\left(y_{j}-y_{j-1}\right)^{2}}\right) .
\end{aligned}
$$

Proof. It is known that the Henstock integrals are additive related to interval. This leads us to

$$
\begin{aligned}
& D\left((F H) \int_{c}^{d}(F H) \int_{a}^{b} f(s, t) d s d t\right. \\
& \left.\sum_{j=1}^{n} \sum_{i=1}^{n}\left(x_{i}-x_{i-1}\right)\left(y_{j}-y_{j-1}\right) \odot f\left(\xi_{i}, \eta_{j}\right)\right) \\
& =D\left((F H) \int_{c}^{d}\left(\sum_{i=1}^{n}(F H) \int_{x_{i-1}}^{x_{i}} f(s, t) d s\right) d t,\right. \\
& =D\left((F H) \sum_{j=1}^{n} \sum_{y_{j-1}}^{n}\left(\sum_{i=1}^{y_{j}}(F H) \int_{x_{i-1}}^{x_{i}} f(s, t) d s\right) d t,\right. \\
& \left.\sum_{j=1}^{n} \sum_{i=1}^{n}(F H) \int_{y_{j-1}}^{y_{j}}(F H) \int_{x_{i-1}}^{x_{i}} f\left(\xi_{i}, \eta_{j}\right) d s d t\right),
\end{aligned}
$$


and, by Definition 3 part (iv) and Theorem 9, we have

$$
\begin{aligned}
& D\left(\sum_{j=1}^{n}(F H) \int_{y_{j-1}}^{y_{j}}\left(\sum_{i=1}^{n}(F H) \int_{x_{i-1}}^{x_{i}} f(s, t) d s\right) d t\right. \\
& \left.\quad \sum_{j=1}^{n} \sum_{i=1}^{n}(F H) \int_{y_{j-1}}^{y_{j}}(F H) \int_{x_{i-1}}^{x_{i}} f\left(\xi_{i}, \eta_{j}\right) d s d t\right) \\
& \leq \sum_{j=1}^{n} D\left((F H) \int_{y_{j-1}}^{y_{j}} \sum_{i=1}^{n}(F H) \int_{x_{i-1}}^{x_{i}} f(s, t) d s d t\right. \\
& \left.\sum_{i=1}^{n}(F H) \int_{y_{j-1}}^{y_{j}}(F H) \int_{x_{i-1}}^{x_{i}} f\left(\xi_{i}, \eta_{j}\right) d s d t\right) \\
& \leq \sum_{j=1}^{n} \sum_{i=1}^{n} D\left((F H) \int_{y_{j-1}}^{y_{j}}(F H) \int_{x_{i-1}}^{x_{i}} f(s, t) d s d t,\right. \\
& \leq \sum_{j=1}^{n} \sum_{i=1}^{n}(L) \int_{y_{j-1}}^{y_{j}}(L) \int_{x_{i-1}}^{x_{i}} D\left(f(s, t), f\left(\xi_{i}, \eta_{j}\right)\right) d s d t . \\
& \left.(F H) \int_{y_{j-1}}^{y_{j}}(F H) \int_{x_{i-1}}^{x_{i}} f\left(\xi_{i}, \eta_{j}\right) d s d t\right)
\end{aligned}
$$

From part (i) of Theorem 6, we conclude that

$$
\begin{gathered}
\sum_{j=1}^{n} \sum_{i=1}^{n}(L) \int_{y_{j-1}}^{y_{j}}(L) \int_{x_{i-1}}^{x_{i}} D\left(f(s, t), f\left(\xi_{i}, \eta_{j}\right)\right) d s d t \\
\leq \sum_{j=1}^{n} \sum_{i=1}^{n}\left(x_{i}-x_{i-1}\right)\left(y_{j}-y_{j-1}\right) \omega_{\left[x_{i-1}, x_{i}\right] \times\left[y_{j-1}, y_{j}\right]} \\
\cdot\left(f, \sqrt{\left(x_{i}-x_{i-1}\right)^{2}+\left(y_{j}-y_{j-1}\right)^{2}}\right),
\end{gathered}
$$

which completes the proof.

From the above inequality, we infer some generalization of well-known trapezoidal-type, midpoint-type, and threepoint-type inequalities with error estimations.

Corollary 18. Assume that $f:[a, b] \times[c, d] \rightarrow R_{F}$ is a Henstock integrable, bounded mapping. Then, with the notation

$$
\omega_{\overline{x y} \times \overline{z t}}=\omega_{[x, y] \times[z, t]}\left(f, \sqrt{(y-x)^{2}+(t-z)^{2}}\right),
$$

one has (i)

$$
\begin{aligned}
& D\left((F H) \int_{c}^{d}(F H) \int_{a}^{b} f(s, t) d s d t\right. \\
& (b-a)(d-c) \odot f(x, y)) \\
& \leq(x-a)(y-c) w_{\overline{a x} \times \overline{c y}}+(b-x)(y-c) w_{\overline{a b} \times \overline{c y}} \\
& +(x-a)(d-y) w_{\overline{a x} \times \overline{y d}} \\
& +(b-x)(d-y) w_{\overline{x b} \times \overline{y d}},
\end{aligned}
$$

(ii)

$$
\begin{aligned}
& D\left((F H) \int_{c}^{d}(F H) \int_{a}^{b} f(s, t) d s d t\right. \\
& {[(x-a)(y-c)} \\
& \quad \odot f(u, \alpha) \oplus(x-a)(d-y) \\
& \quad \odot f(u, \beta) \oplus(b-x)(d-y) \\
& \quad \odot f(c, \beta) \oplus(b-x)(d-y) \\
& \quad \odot f(v, \beta)]) \\
& \leq \quad(x-a)(y-c) w_{\overline{a x} \times \overline{c y}}+(b-x)(y-c) w_{\overline{x b} \times \overline{c y}} \\
& +(x-a)(d-y) w_{\overline{a x} \times \overline{y d}} \\
& +(b-x)(d-y) w_{\overline{x b} \times \overline{y d}},
\end{aligned}
$$

for any $x \in[a, b], y \in[c, d], u \in[a, x], v \in[x, b], \alpha \in[c, y]$, and $\beta \in[y, d]$;

(iii)

$$
\begin{gathered}
D\left((F H) \int_{c}^{d}(F H) \int_{a}^{b} f(s, t) d s d t,\right. \\
{[(\alpha-a)(\theta-c) \odot f(u, r)} \\
\oplus(\alpha-a)(\gamma-\theta) \odot f(u, p) \\
\oplus(\alpha-a)(d-\gamma) \odot f(u, z) \\
\oplus(\beta-\alpha)(\theta-c) \odot f(\nu, r) \\
\oplus(\beta-\alpha)(\gamma-\theta) \odot f(\nu, p)
\end{gathered}
$$




$$
\begin{aligned}
& \oplus(\beta-\alpha)(d-\gamma) \odot f(u, z) \\
& \oplus(b-\beta)(\theta-c) \odot f(w, r) \\
& \oplus(b-\beta)(\gamma-\theta) \odot f(w, p) \\
& +(b-\beta)(d-\gamma) \odot f(w, z)]) \\
& \leq(\alpha-a)(\theta-c) \omega_{\overline{a \alpha} \times \overline{c \theta}}+(\alpha-a)(\gamma-\theta) \omega_{\overline{a \alpha} \times \overline{\theta \gamma}} \\
& +(\alpha-a)(d-\gamma) \omega_{\overline{a \alpha} \times \overline{\gamma d}} \\
& +(\beta-\alpha)(\theta-c) \omega_{\overline{\alpha \beta} \times \overline{c \theta}}+(\beta-\alpha)(\gamma-\theta) \omega_{\overline{\alpha \beta} \times \overline{\theta \gamma}} \\
& +(\beta-\alpha)(d-\gamma) \omega_{\overline{\alpha \beta} \times \overline{\gamma d}}+(b-\beta)(\theta-c) \omega_{\overline{\beta b} \times \overline{c \theta}} \\
& +(b-\beta)(\gamma-\theta) \omega_{\overline{\beta b} \times \overline{\theta \gamma}}+(b-\beta)(d-\gamma) \omega_{\overline{\beta b} \times \overline{\gamma d}}
\end{aligned}
$$

for any $u, v, w, \alpha, \beta, r, p, \theta, \gamma$, and $z$ with $a<u<\alpha<v<$ $\beta<w<b$ and $c<r<\theta<p<\gamma<z<d$.

Proof. (i) Taking in the previous theorem that $n=2, x_{1}=$ $\xi_{1}=\xi_{2}=x$, and $y_{1}=\eta_{1}=\eta_{2}=y$, we obtain the required inequality. Indeed,

$$
\begin{aligned}
& D\left((F H) \int_{c}^{d}(F H) \int_{a}^{b} f(s, t) d s d t\right. \\
& \left.\sum_{j=1}^{2} \sum_{i=1}^{2}\left(x_{i}-x_{i-1}\right)\left(y_{j}-y_{j-1}\right) \odot f\left(\xi_{i}, \eta_{j}\right)\right) \\
& =D\left((F H) \int_{c}^{d}(F H) \int_{a}^{b} f(s, t) d s d t,\right. \\
& \sum_{j=1}^{2}\left[(x-a)\left(y_{j}-y_{j-1}\right) \odot f(x, y)\right. \\
& \left.\left.\oplus(b-x)\left(y_{j}-y_{j-1}\right) \odot f\left(x, \eta_{j}\right)\right]\right) \\
& =D\left((F H) \int_{c}^{d}(F H) \int_{a}^{b} f(s, t) d s d t\right. \\
& \sum_{j=1}^{2}[(x-a)(y-c) \odot f(x, y) \\
& \oplus(b-x)(y-c) \odot f(x, y) \\
& \oplus(x-a)(d-y) \odot f(x, y) \\
& \oplus(b-x)(d-y) \odot f(x, y)])
\end{aligned}
$$

$$
\begin{aligned}
& =D\left((F H) \int_{c}^{d}(F H) \int_{a}^{b} f(s, t) d s d t\right. \\
& \left.\quad \sum_{j=1}^{2}[(b-a)(d-c) \odot f(x, y)]\right) \\
& \leq \sum_{j=1}^{n} \sum_{i=1}^{n}\left(x_{i}-x_{i-1}\right)\left(y_{j}-y_{j-1}\right) \omega_{\left[x_{i-1}, x_{i}\right] \times\left[y_{j-1}, y_{j}\right]} \\
& \quad \cdot\left(f, \sqrt{\left(x_{i}-x_{i-1}\right)^{2}+\left(y_{j}-y_{j-1}\right)^{2}}\right) \\
& =(x-a)(y-c) \omega_{\overline{a x} \times \overline{c y}}+(b-x)(y-c) \omega_{\overline{x b} \times \overline{c y}} \\
& \quad+(x-a)(d-y) \omega_{\overline{a x} \times \overline{y d}}+(b-x)(d-y) \omega_{\overline{x b} \times \overline{y d}} .
\end{aligned}
$$

(ii) Taking that $n=2, x_{1}=x, \xi_{1}=u, \xi_{2}=v, y_{1}=y$, $\eta_{1}=\alpha$, and $\eta_{2}=\beta$ in Theorem 17, we obtain the required inequality. Indeed,

$$
\begin{aligned}
& D\left((F H) \int_{c}^{d}(F H) \int_{a}^{b} f(s, t) d s d t\right. \\
& \left.\sum_{j=1}^{2} \sum_{i=1}^{2}\left(x_{i}-x_{i-1}\right)\left(y_{j}-y_{j-1}\right) \odot f\left(\xi_{i}, \eta_{j}\right)\right) \\
& \leq D\left((F H) \int_{c}^{d}(F H) \int_{a}^{b} f(s, t) d s d t\right. \\
& \sum_{j=1}^{2}\left[(x-a)\left(y_{j}-y_{j-1}\right) \odot f\left(u, \eta_{j}\right)\right. \\
& \left.\left.\oplus(b-x)\left(y_{j}-y_{j-1}\right) \odot f\left(v, \eta_{j}\right)\right]\right) \\
& =D\left((F H) \int_{c}^{d}(F H) \int_{a}^{b} f(s, t) d s d t\right. \\
& {[(x-a)(y-c) \odot f(u, \alpha)} \\
& \oplus(x-a)(d-y) \odot f(u, \beta) \\
& \oplus(b-x)(y-c) \odot f(v, \alpha) \\
& \oplus(b-x)(d-y) \odot f(\nu, \beta)]) \\
& \leq \sum_{j=1}^{2} \sum_{i=1}^{2}\left(x_{i}-x_{i-1}\right)\left(y_{j}-y_{j-1}\right) \omega_{\overline{x_{i-1}, x_{i}} \times \overline{y_{j-1}}, y_{j}} \\
& =(x-a)(y-c) \omega_{\overline{a x} \times \overline{c y}}+(b-x)(y-c) \omega_{\overline{x b} \times \overline{c y}} \\
& +(x-a)(d-y) \omega_{\overline{a x} \times \overline{y d}}+(b-x)(d-y) \omega_{\overline{x b} \times \overline{y d}} .
\end{aligned}
$$


(iii) Considering $n=4$ and performing the similar way in part (ii), it is obvious that the inequality in previous theorem becomes the inequality stated above.

Corollary 19. Let $f:[a, b] \times[c, d] \rightarrow R_{F}$ be a twodimensional Henstock integrable, bounded mapping. Then, the following inequalities hold:

(i) $D\left((F H) \int_{c}^{d}(F H) \int_{a}^{b} f(s, t) d s d t\right.$

$$
\begin{aligned}
& \left.(b-a)(d-c) \odot f\left(\frac{a+b}{2}, \frac{c+d}{2}\right)\right) \\
& \leq(b-a)(d-c) w_{[a, b] \times[c, d]}\left(f, \frac{(b-a)(d-c)}{4}\right),
\end{aligned}
$$

(ii) $D\left((F H) \int_{c}^{d}(F H) \int_{a}^{b} f(s, t) d s d t\right.$,

$$
\begin{aligned}
\frac{(b-a)(d-c)}{4} \odot[ & f(a, c) \oplus f(a, d) \\
& \oplus f(b, c) \oplus f(b, d)])
\end{aligned}
$$$$
\leq(b-a)(d-c) w_{[a, b] \times[c, d]}\left(f, \frac{(b-a)(d-c)}{4}\right),
$$

(iii) $D\left((F H) \int_{a}^{b} f(s, t) d s d t\right.$,

$$
\begin{aligned}
& \frac{(b-a)(d-c)}{36} \odot[ f(a, c) \oplus f(a, d) \\
& \oplus 4 \odot f\left(a, \frac{c+d}{2}\right) \\
& \oplus 4 \odot f\left(\frac{a+b}{2}, c\right) \\
& \oplus 16 \odot f\left(\frac{a+b}{2}, \frac{a+c}{2}\right) \\
& \oplus 4 \odot f\left(\frac{a+b}{2}, d\right) \\
& \oplus 4 \odot f\left(b, \frac{c+d}{2}\right) \\
&\oplus f(b, c) \oplus f(b, d)]) \\
& \leq(b-a)(d-c) w_{[a, b] \times[c, d]}\left(f, \frac{(b-a)(d-c)}{36}\right) .
\end{aligned}
$$

Proof. (i) If we take $x=(a+b) / 2$ and $y=(c+d) / 2$ in the assertion (i) of Corollary 18, we obtain the required inequality. In other words, we have

$$
\begin{aligned}
D( & (F H) \int_{c}^{d}(F H) \int_{a}^{b} f(s, t) d s d t, \\
& \left.(b-a)(d-c) \odot f\left(\frac{a+b}{2}, \frac{c+d}{2}\right)\right) \\
\leq & \left(\frac{a+b}{2}-a\right)\left(\frac{c+d}{2}-c\right) \omega_{\overline{a((a+b) / 2)} \times \overline{c((c+d) / 2)}} \\
& +\left(b-\frac{a+b}{2}\right)\left(\frac{c+d}{2}-c\right) \omega_{\overline{((a+b) / 2) b \times c((c+d) / 2)}} \\
& +\left(\frac{a+b}{2}-a\right)\left(d-\frac{c+d}{2}\right) \omega_{\overline{a((a+b) / 2)} \times \overline{((c+d) / 2) d}} \\
& +\left(b-\frac{a+b}{2}\right)\left(d-\frac{c+d}{2}\right) \omega_{\overline{((a+b) / 2) b \times} \times \overline{((c+d) / 2) d}} \\
= & \frac{(b-a)(d-c)}{4} \\
& \times\left[\omega_{\overline{a((a+b) / 2)} \times \overline{c((c+d) / 2)}}+\omega_{\overline{((a+b) / 2) b \times c} \times \overline{c((c+d) / 2)}}\right. \\
& \left.+\omega_{\overline{a((a+b) / 2)} \times \overline{((c+d) / 2) d}}+\omega_{\overline{((a+b) / 2) b} \times \overline{((c+d) / 2) d}}\right] \\
\leq & (b-a)(d-c) \omega_{[a, b] \times[c, d]}\left(f, \frac{(b-a)(d-c)}{4}\right) .
\end{aligned}
$$

(ii) Taking $x=(a+b) / 2, y=(c+d) / 2, u=a, v=b, \alpha=c$, and $\beta=d$ in the assertion (ii) of the previous corollary, we obtain

$$
\begin{aligned}
& D\left((F H) \int_{c}^{d}(F H) \int_{a}^{b} f(s, t) d s d t,\right. \\
& \left(\frac{a+b}{2}-a\right)\left(\frac{c+d}{2}-c\right) \odot f(a, c) \\
& \oplus\left(\frac{a+b}{2}-a\right)\left(d-\frac{c+d}{2}\right) \odot f(a, d) \\
& \oplus\left(b-\frac{a+b}{2}\right)\left(\frac{c+d}{2}-c\right) \odot f(b, c) \\
& \left.\oplus\left(b-\frac{a+b}{2}\right)\left(d-\frac{c+d}{2}\right) \odot f(a, d)\right) \\
& =\frac{(b-a)(d-c)}{4} \\
& \times\left[\omega_{\overline{a((a+b) / 2)} \times \overline{c((c+d) / 2)}}+\omega_{\overline{((a+b) / 2) b} \times \overline{c((c+d) / 2)}}\right. \\
& \left.+\omega_{\overline{a((a+b) / 2)} \times \overline{((c+d) / 2) d}}+\omega_{\overline{((a+b) / 2) b} \times \overline{((c+d) / 2) d}}\right] \\
& \leq(b-a)(d-c) \omega_{[a, b] \times[c, d]}\left(f, \frac{(b-a)(d-c)}{4}\right) .
\end{aligned}
$$

(iii) It is easy to see that the inequality follows from the corresponding assertion (iii) of the previous corollary by 
taking $n=4, \alpha=(5 a+b) / 6, \beta=(a+5 b) / 6, u=a$, $v=(a+b) / 2, w=b, \theta=(5 c+d) / 6, \gamma=(c+5 d) / 6, r=c$, $p=(c+d) / 2$, and $z=d$. Indeed, we have

$$
\begin{aligned}
& D\left((F H) \int_{c}^{d}(F H) \int_{a}^{b} f(s, t) d s d t\right. \\
& \frac{(b-a)(d-c)}{36} \\
& \odot\left[f(a, c) \oplus f(a, d) \oplus 4 \odot f\left(a, \frac{c+d}{2}\right)\right. \\
& \oplus 4 \odot f\left(\frac{a+b}{2}, c\right) \\
& \oplus 16 \odot f\left(\frac{a+b}{2}, \frac{a+c}{2}\right) \oplus 4 \odot f\left(\frac{a+b}{2}, d\right) \\
& \left.\left.\oplus 4 \odot f\left(b, \frac{c+d}{2}\right) \oplus f(b, c) \oplus f(b, d)\right]\right) \\
& \leq(b-a)(d-c) \omega_{[a, b] \times[c, d]}\left(f, \frac{(b-a)(d-c)}{36}\right) .
\end{aligned}
$$

The next corollaries present simpler error estimation for the inequality stated in Theorem 17.

Corollary 20. Let $f:[a, b] \times[c, d] \rightarrow R_{F}$ be a twodimensional Henstock integrable, bounded mapping. Then, for any divisions $\Delta_{x}^{n}: a=x_{0}<x_{1}<\cdots<x_{n}=b$ and $\Delta_{y}^{n}: c=y_{0}<y_{1}<\cdots<y_{n}=d, \xi_{i} \in\left[x_{i-1}, x_{i}\right]$ and $\eta_{i} \in\left[y_{j-1}, y_{j}\right], i=1, \ldots, n ; j=1, \ldots, n$, one has

$$
\begin{aligned}
& D\left((F H) \int_{c}^{d}(F H) \int_{a}^{b} f(s, t) d s d t\right. \\
& \left.\quad \sum_{j=1}^{n} \sum_{i=1}^{n}\left(x_{i}-x_{i-1}\right)\left(y_{j}-y_{j-1}\right) \odot f\left(\xi_{i}, \eta_{j}\right)\right) \\
& \leq(b-a)(d-c) \omega_{[a, b] \times[c, d]}\left(f, v\left(\Delta_{x y}\right)\right),
\end{aligned}
$$

where $v\left(\Delta_{x y}\right)=\max _{i, j=1, \ldots, n}\left\{\left(x_{i}-x_{i-1}\right)\left(y_{j}-y_{j-1}\right)\right\}$ is the norm of the divisions $\Delta_{x}^{n}$ and $\Delta_{y}^{n}$.

Proof. Considering Theorem 17 and parts (i), (ii) of Theorem 13 and by regarding the definition of $\bigvee(f)$, we infer that

$$
\begin{aligned}
D( & (F H) \int_{c}^{d}(F H) \int_{a}^{b} f(s, t) d s d t \\
& \left.\sum_{j=1}^{n} \sum_{i=1}^{n}\left(x_{i}-x_{i-1}\right)\left(y_{j}-y_{j-1}\right) \odot f\left(\xi_{i}, \eta_{j}\right)\right) \\
\leq & \sum_{j=1}^{n} \sum_{i=1}^{n}\left(x_{i}-x_{i-1}\right)\left(y_{j}-y_{j-1}\right) \omega_{\left[x_{i-1}, x_{i}\right] \times\left[y_{j-1}, y_{j}\right]} \\
& \cdot\left(f, \sqrt{\left(x_{i}-x_{i-1}\right)^{2}+\left(y_{j}-y_{j-1}\right)^{2}}\right)
\end{aligned}
$$

$$
\begin{aligned}
& \leq \sum_{j=1}^{n} \sum_{i=1}^{n}\left(x_{i}-x_{i-1}\right)\left(y_{j}-y_{j-1}\right) \omega_{[a, b] \times[c, d]}\left(f, v\left(\Delta_{x y}\right)\right) \\
& =(b-a)(c-d) \omega_{[a, b] \times[c, d]}\left(f, v\left(\Delta_{x y}\right)\right) .
\end{aligned}
$$

Corollary 21. Let $f:[a, b] \times[c, d] \rightarrow R_{F}$ be a twodimensional Henstock integrable, bounded mapping. Then, for any divisions $\Delta_{x}^{n}: a=x_{0}<x_{1}<\cdots<x_{n}=b$ and $\Delta_{y}^{n}: c=y_{0}<y_{1}<\cdots<y_{n}=d, \xi_{i} \in\left[x_{i-1}, x_{i}\right]$ and $\eta_{i} \in\left[y_{j-1}, y_{j}\right], i=1, \ldots, n ; j=1, \ldots, n$, one has

$$
\begin{aligned}
& D\left((F H) \int_{c}^{d}(F H) \int_{a}^{b} f(s, t) d s d t\right. \\
& \left.\quad \sum_{j=1}^{n} \sum_{i=1}^{n}\left(x_{i}-x_{i-1}\right)\left(y_{j}-y_{j-1}\right) \odot f\left(\xi_{i}, \eta_{j}\right)\right) \\
& \leq v\left(\Delta_{x y}\right) \sum_{j=1}^{n} \sum_{i=1}^{n} \omega_{[a, b] \times[c, d]} \\
& \cdot\left(f, \sqrt{\left(x_{i}-x_{i-1}\right)^{2}+\left(y_{j}-y_{j-1}\right)^{2}}\right) .
\end{aligned}
$$

Proof. Since $v\left(\Delta_{x y}\right)$ is the least upper bound of partitions $\Delta_{x}^{n}$ and $\Delta_{y}^{n}$, we conclude that $\left(x_{i}-x_{i-1}\right)\left(y_{j}-y_{j-1}\right) \leq v\left(\Delta_{x y}\right)$ for any $i, j=\overline{1, n}$. Hence, the required inequality holds.

Remark 22. If $f:[a, b] \times[c, d] \rightarrow R_{F}$ is a two-dimensional Riemann integrable function, it is also Henstock integrable function. Therefore, the above quadrature rules hold for Riemann integrable function too.

Theorem 23. Let $f:[a, b] \times[c, d] \rightarrow R_{F}$ be a mapping of bounded variation. Then, for any divisions $\Delta_{x}^{n}: a=x_{0}<$ $x_{1}<\cdots<x_{n}=b$ and $\Delta_{y}^{n}: c=y_{0}<y_{1}<\cdots<y_{n}=d$, $\xi_{i} \in\left[x_{i-1}, x_{i}\right]$ and $\eta_{i} \in\left[y_{j-1}, y_{j}\right], i=1, \ldots, n ; j=1, \ldots, n$, one has

$$
\begin{aligned}
& D\left((F R) \int_{c}^{d}(F R) \int_{a}^{b} f(s, t) d s d t\right. \\
& \left.\quad \sum_{j=1}^{n} \sum_{i=1}^{n}\left(x_{i}-x_{i-1}\right)\left(y_{j}-y_{j-1}\right) \odot f\left(\xi_{i}, \eta_{j}\right)\right) \\
& \leq v\left(\Delta_{x y}\right) \cdot \bigvee(f) .
\end{aligned}
$$

Proof. If we define $\varphi_{x y}:[a, b] \times[c, d] \rightarrow R_{+}$such that $\varphi_{x y}(s, t)=D(f(s, t), f(x, y))$ for any $(x, y) \in[a, b] \times[c, d]$, we see that $\varphi$ is of bounded variation and we have

$$
\bigvee \varphi_{x y}(s, t) \leq \bigvee(f), \quad(x, y) \in[a, b] \otimes[c, d]
$$


in other words,

$$
\begin{aligned}
& V_{\Delta_{x y}^{n}}(D(f(s, t), f(x, y))) \\
& =\sum_{k=0}^{n-1} \sum_{m=0}^{n-1} \mid D\left(f\left(t_{k+1}, s_{m+1}\right), f(x, y)\right) \\
& -D\left(f\left(t_{k}, s_{m}\right), f(x, y)\right) \\
& \leq \sum_{k=0}^{n-1} \sum_{m=0}^{n-1}\left(D\left(f\left(t_{k+1}, s_{m+1}\right), f(x, y)\right)\right)=V_{\Delta_{x y}^{n}}(f) \text {. }
\end{aligned}
$$

Considering Theorem 13, Theorem 17, Corollary 21, and [18] and since any real valued function of bounded variation is Lebesgue integrable, we observe that

$$
\begin{aligned}
\sum_{j=1}^{n} \sum_{i=1}^{n}\left(x_{i}-x_{i-1}\right)\left(y_{j}-y_{j-1}\right) \omega_{\left[x_{i-1}, x_{i}\right] \times\left[y_{j-1}, y_{j}\right]} & \cdot\left(f, \sqrt{\left(x_{i}-x_{i-1}\right)^{2}+\left(y_{j}-y_{j-1}\right)^{2}}\right) \\
\leq & v\left(\Delta_{x y}\right) \sum_{j=1}^{n} \sum_{i=1}^{n} \omega_{\left[x_{i-1}, x_{i}\right] \times\left[y_{j-1}, y_{j}\right]} \\
& \cdot\left(f, \sqrt{\left(x_{i}-x_{i-1}\right)^{2}+\left(y_{j}-y_{j-1}\right)^{2}}\right) \\
= & v\left(\Delta_{x y}\right) \sum_{j=1}^{n} \sum_{i=1}^{n} \bigvee_{\left[y_{j-1}, x_{j}\right]}(f) \\
= & v\left(\Delta_{x y}\right) \cdot \bigvee(f) .
\end{aligned}
$$

Theorem 24. If $f:[a, b] \times[c, d] \rightarrow R_{F}$ is L-Lipschitz mapping, then, for any divisions $\Delta_{x}^{n}: a=x_{0}<x_{1}<\cdots<$ $x_{n}=b$ and $\Delta_{y}^{n}: c=y_{0}<y_{1}<\cdots<y_{n}=d, \xi_{i} \in\left[x_{i-1}, x_{i}\right]$ and $\eta_{i} \in\left[y_{j-1}, y_{j}\right], i=1, \ldots, n ; j=1, \ldots, n$, one has

$$
\begin{aligned}
& D\left((F R) \int_{c}^{d}(F R) \int_{a}^{b} f(s, t) d s d t\right. \\
& \left.\quad \sum_{j=1}^{n} \sum_{i=1}^{n}\left(x_{i}-x_{i-1}\right)\left(y_{j}-y_{j-1}\right) \odot f\left(\xi_{i}, \eta_{j}\right)\right) \\
& \leq L \sum_{j=1}^{n} \sum_{i=1}^{n}\left(\left(x_{i}-x_{i-1}\right)^{2}\left(y_{j}-y_{j-1}\right)^{2}\right) .
\end{aligned}
$$

Proof. Analogous to the proof of Theorem 17 and by definition of L-Lipschitz mapping, we infer that

$$
\begin{aligned}
& D\left((F R) \int_{c}^{d}(F R) \int_{a}^{b} f(s, t) d s d t,\right. \\
& \left.\quad \sum_{j=1}^{n} \sum_{i=1}^{n}\left(x_{i}-x_{i-1}\right)\left(y_{j}-y_{j-1}\right) \odot f\left(\xi_{i}, \eta_{j}\right)\right) \\
& \leq \sum_{j=1}^{n} \sum_{i=1}^{n}(L) \int_{y_{j-1}}^{y_{j}}(L) \int_{x_{i-1}}^{x_{i}} D\left(f(s, t), f\left(\xi_{i}, \eta_{j}\right)\right) d s d t \\
& \leq L \sum_{j=1}^{n} \sum_{i=1}^{n}(L) \int_{y_{j-1}}^{y_{j}}(L) \int_{x_{i-1}}^{x_{i}} \sqrt{\left(s-\xi_{i}\right)^{2}+\left(t-\eta_{j}\right)^{2}} d s d t \\
& \leq L \sum_{j=1}^{n} \sum_{i=1}^{n}(L) \int_{y_{j-1}}^{y_{j}}(L) \\
& \cdot \sum_{j=1}^{x_{i}}\left(\left(x_{i}-x_{i-1}\right)^{2}\right. \\
& =L \sum_{x_{i-1}}^{n}\left(\left(x_{i}-x_{i-1}\right)^{2}+\left(y_{j}-y_{j-1}\right)^{2}\right) .
\end{aligned}
$$

\section{2D Fuzzy Fredholm Integral Equations}

Here, we consider the two-dimensional fuzzy Fredholm integral equations as follows:

$$
F(s, t)=f(s, t) \oplus \lambda \odot \int_{c}^{d} \int_{a}^{b} K(s, t, x, y) \odot F(x, y) d x d y,
$$

where $\lambda>0, K(s, t, x, y)$ is an arbitrary positive kernel on $[a, b] \times[c, d] \times[a, b] \times[c, d]$ and $f:[a, b] \times[c, d] \rightarrow R_{F}$. We assume that $K$ is continuous, and therefore it is uniformly continuous with respect to $(s, t)$. This property implies that there exists $M>0$ such that

$$
M=\max _{\substack{a \leq s, x \leq b \\ c \leq t, y \leq d}}|K(s, t, x, y)|
$$

Now, we will prove the existence and uniqueness of the solution of (41) by the method of successive approximations. Let $X=\left\{f:[a, b] \times[c, d] \rightarrow R_{F} ; f\right.$ is continuous $\}$ be the space of two-dimensional fuzzy continuous functions with the metric

$$
D^{*}(f, g)=\sup _{\substack{a \leq s \leq b \\ c \leq t \leq d}} D(f(s, t), g(s, t))
$$


that is called the uniform distance between two-dimensional fuzzy-number-valued functions. We define the operator $A$ : $X \rightarrow X$ by

$$
\begin{array}{r}
A(F)(s, t) \\
=f(s, t) \oplus \lambda \odot \int_{c}^{d} \int_{a}^{b} K(s, t, x, y) \odot F(x, y) d x d y, \\
\forall(s, t) \in[a, b] \times[c, d], \quad \forall f \in X .
\end{array}
$$

Sufficient conditions for the existence of a unique solution of (41) are given in the following result.

Theorem 25. Let $K(s, t, x, y)$ be continuous and positive for $a \leq s, x \leq b$, and $c \leq t, y \leq d$, and let $f:[a, b] \times[c, d] \rightarrow R_{F}$ be continuous on $[a, b] \times[c, d]$. If $B=\lambda M(b-a)(d-c)<1$, then the iterative procedure

$$
\begin{aligned}
F_{0}(s, t)= & f(s, t), \\
F_{m}(s, t)= & f(s, t) \oplus \lambda \odot(F R) \\
& \cdot \int_{c}^{d}(F R) \int_{a}^{b} K(s, t, x, y) \odot F_{m-1}(x, y) d x d y,
\end{aligned}
$$$$
m \geq 1 \text {, }
$$

converges to the unique solution $F^{*}$ of (41).

Moreover, the following error bound holds:

$$
D^{*}\left(F^{*}, F_{m}\right) \leq \frac{B^{m+1}}{1-B} M_{1},
$$

where

$$
M_{1}=\sup _{\substack{a \leq s \leq b \\ c \leq t \leq d}}\|F(s, t)\|_{F} .
$$

Proof. To prove this theorem, we investigate the conditions of the Banach fixed point principle. We first show that $A$ maps $X$ into $X$ (i.e., $A(X) \subset X$ ). To the end, we show that the operator $A$ is uniformly continuous. Since $f$ is continuous on compact set of $[a, b] \times[c, d]$, we deduce that it is uniformly continuous, and hence for $\varepsilon_{1}>0$ exists $\delta_{1}>0$ such that

$$
\begin{aligned}
& D\left(f\left(s_{1}, t_{1}\right), f\left(s_{2}, t_{2}\right)\right)<\varepsilon_{1} \\
& \text { whenever } \sqrt{\left(t_{2}-t_{1}\right)^{2}+\left(s_{2}-s_{1}\right)^{2}}<\delta_{1}, \\
& \qquad \forall s_{1}, s_{2} \in[a, b], \quad \forall t_{1}, t_{2} \in[c, d] .
\end{aligned}
$$

As mentioned above, $K$ also is uniformly continuous; thus, for $\varepsilon_{2}>0$ exists $\delta_{2}>0$ such that

$$
\begin{aligned}
& \left|K\left(s_{1}, t_{1}, x, y\right)-K\left(s_{2}, t_{2}, x, y\right)\right|<\varepsilon_{2} \\
& \text { whenever } \sqrt{\left(t_{2}-t_{1}\right)^{2}+\left(s_{2}-s_{1}\right)^{2}}<\delta_{2}, \\
& \forall s_{1}, s_{2} \in[a, b], \quad \forall t_{1}, t_{2} \in[c, d] .
\end{aligned}
$$

Let $\delta=\min \left\{\delta_{1}, \delta_{2}\right\}, s_{1}, s_{2} \in[a, b]$, and $t_{1}, t_{2} \in[c, d]$, with $\sqrt{\left(t_{2}-t_{1}\right)^{2}+\left(s_{2}-s_{1}\right)^{2}}<\delta$. According to Definition 3 and Theorem 9 , we obtain

$$
\begin{aligned}
& D\left(A(F)\left(s_{1}, t_{1}\right), A(F)\left(s_{2}, t_{2}\right)\right) \\
& \leq D\left(f\left(s_{1}, t_{1}\right), f\left(s_{2}, t_{2}\right)\right) \\
& +D\left(\lambda \odot(F R) \int_{c}^{d}(F R) \int_{a}^{b} K\left(s_{1}, t_{1}, x, y\right)\right. \\
& \odot F(x, y) d x d y, \\
& \lambda \odot(F R) \int_{c}^{d}(F R) \int_{a}^{b} K\left(s_{2}, t_{2}, x, y\right) \\
& \odot F(x, y) d x d y) \\
& \leq \varepsilon_{1}+\lambda \odot(F R) \int_{c}^{d} D\left((F R) \int_{a}^{b} K\left(s_{1}, t_{1}, x, y\right)\right. \\
& \odot F(x, y) d x, \\
& \text { (FR) } \int_{a}^{b} K\left(s_{2}, t_{2}, x, y\right) \\
& \odot F(x, y) d x) d y \\
& \leq \varepsilon_{1}+\lambda \odot(F R) \\
& \cdot \int_{c}^{d}(F R) \int_{a}^{b} D\left(K\left(s_{1}, t_{1}, x, y\right) \odot F(x, y),\right. \\
& \left.K\left(s_{2}, t_{2}, x, y\right) \odot F(x, y)\right) d x d y \\
& \leq \varepsilon_{1}+\lambda\left|K\left(s_{1}, t_{1}, x, y\right)-K\left(s_{2}, t_{2}, x, y\right)\right| \\
& \odot(F R) \int_{c}^{d}(F R) \int_{a}^{b}(F(x, y), \widetilde{0}) d x d y \\
& \leq \varepsilon_{1}+\lambda \varepsilon_{2} \odot(F R) \int_{c}^{d}(F R) \int_{a}^{b}\|F(x, y)\|_{F} d x d y \\
& \leq \varepsilon_{1}+\lambda \varepsilon_{2}(b-a)(d-c)\|F(x, y)\|_{F} \\
& \leq \varepsilon_{1}+\lambda(b-a)(d-c) M_{1} \varepsilon_{2} \\
& \text { where } M_{1}=\sup _{\substack{a \leq s \leq b \\
c \leq t \leq d}}\|F(s, t)\|_{F} \text {, }
\end{aligned}
$$

and by choosing $\varepsilon_{1}=\varepsilon / 2$ and $\varepsilon_{2}=\left(1 / 2 M_{1} \lambda(b-a)(d-c)\right) \varepsilon$, we derive

$$
D\left(A(F)\left(s_{1}, t_{1}\right), A(F)\left(s_{2}, t_{2}\right)\right) \leq \varepsilon .
$$

This shows that $A(F)$ is uniformly continuous for any $F \in$ $X$ and so continuous on $[a, b] \times[c, d]$, and hence $A(X) \subset X$. 
Now, we prove that the operator $A$ is a contraction map. So, for $F_{1}, F_{2} \in X, s \in[a, b]$, and $t \in[c, d]$, we have

$$
\begin{aligned}
& D\left(A\left(F_{1}\right)(s, t), A\left(F_{2}\right)(s, t)\right) \\
& \leq D(f(s, t), f(s, t)) \\
& +D\left(\lambda \odot(F R) \int_{c}^{d}(F R) \int_{a}^{b} K(s, t, x, y)\right. \\
& \odot F_{1}(x, y) d x d y \text {, } \\
& \lambda \odot(F R) \int_{c}^{d}(F R) \int_{a}^{b} K(s, t, x, y) \\
& \left.\odot F_{2}(x, y) d x d y\right) \\
& \leq \lambda \odot(F R) \int_{c}^{d}(F R) \int_{a}^{b} D\left(K(s, t, x, y) \odot F_{1}(x, y),\right. \\
& K(s, t, x, y) \\
& \left.\odot F_{2}(x, y)\right) d x d y \\
& =\lambda|K(s, t, x, y)| \odot(F R) \\
& \cdot \int_{c}^{d}(F R) \int_{a}^{b} D\left(F_{1}(x, y), F_{2}(x, y)\right) d x d y \\
& \leq \lambda M \odot(F R) \int_{c}^{d}(F R) \int_{a}^{b} D\left(F_{1}(x, y),\right. \\
& \left.F_{2}(x, y)\right) d x d y \\
& \leq \lambda M \odot(F R) \int_{c}^{d}(F R) \int_{a}^{b} D^{*}\left(F_{1}, F_{2}\right) d x d y \\
& =\lambda M(b-a)(d-c) D^{*}\left(F_{1}, F_{2}\right)=B D^{*}\left(F_{1}, F_{2}\right) \text {. }
\end{aligned}
$$

Therefore, we obtained

$$
D^{*}\left(A\left(F_{1}\right)(s, t), A\left(F_{2}\right)(s, t)\right) \leq B D^{*}\left(F_{1}, F_{2}\right) .
$$

Since $B<1$, the operator $A$ is a contraction on Banach space $\left(X, D^{*}\right)$. Consequently, Banach's fixed point principle implies that (41) has a unique solution $F^{*}$ in $X$ and we also have

$$
\begin{aligned}
D & \left(F^{*}(s, t), F_{m}(s, t)\right) \\
& \leq D^{*}\left(F^{*}, F_{m}\right) \\
& \leq \lambda M(b-a)(d-c) D^{*}\left(F^{*}, F_{m-1}\right) \\
& =B D^{*}\left(F^{*}, F_{m-1}\right) \leq B D^{*}\left(F^{*}, F_{m}\right)+B D^{*}\left(F_{m-1}, F_{m}\right) \\
& \leq B D^{*}\left(F^{*}, F_{m}\right)+B^{m} D^{*}\left(F_{0}, F_{1}\right) ;
\end{aligned}
$$

therefore,

$$
D^{*}\left(F^{*}, F_{m}\right) \leq \frac{B^{m}}{1-B} D^{*}\left(F_{0}, F_{1}\right)
$$

on the other hand,

$$
\begin{aligned}
& D^{*}\left(F_{0}, F_{1}\right) \\
& =\sup _{\substack{a \leq s \leq b \\
c \leq t \leq d}} D(f(s, t) \oplus \widetilde{0}, f(s, t) \oplus \lambda \otimes(F R) \\
& \cdot \cdot \int_{c}^{d}(F R) \int_{a}^{b} K(s, t, x, y) \\
& \leq \sup _{a \leq s \leq b} \lambda \odot(F R) \\
& \left.\quad F_{c \leq t \leq d}(x, y) d x d y\right) \\
& \quad \cdot \int_{c}^{d}(F R) \int_{a}^{b} D\left(\widetilde{0}, K(s, t, x, y) \odot F_{0}(x, y) d x d y\right) \\
& \leq M_{c} \odot(F R) \int_{c}^{d}(F R) \int_{a}^{b} \sup _{a \leq s \leq b} D\left(\widetilde{0}, F_{0}(x, y)\right) d x d y \\
& =\lambda M_{c \leq t \leq d}(b-a)(d-c) M_{1}=M_{1} B,
\end{aligned}
$$

so by (55) and (56), we obtained inequality (46), which completes the proof.

Now, we introduce a numerical method to solve (41). We consider (41) with continuous kernel $K(s, t, x, y)$ having positive sign on $[a, b] \times[c, d] \times[a, b] \times[c, d]$ and uniform partitions

$$
\begin{aligned}
& D_{x}: a=s_{0}<s_{1}<s_{2}<\cdots<s_{n-1}<s_{n}=b, \\
& D_{y}: b=t_{0}<t_{1}<t_{2}<\cdots<t_{n-1}<t_{n}=d,
\end{aligned}
$$

with $s_{i}=a+i h, t_{j}=c+j h^{\prime}$, where $h=(b-a) / n, h^{\prime}=(d-c) / n$. Then, the following iterative procedure gives the approximate solution of $(41)$ in point $(s, t)$ :

$$
\begin{aligned}
& u_{0}(s, t)= f(s, t), \\
& u_{m}(s, t)= f(s, t) \oplus \frac{\lambda h h^{\prime}}{4} \\
& \odot\left[\left(K\left(s, t, s_{0}, t_{0}\right) \odot u_{m-1}\left(s_{0}, t_{0}\right)\right.\right. \\
& \oplus K\left(s, t, s_{0}, t_{n}\right) \odot u_{m-1}\left(s_{0}, t_{n}\right) \\
& \oplus K\left(s, t, s_{n}, t_{0}\right) \odot u_{m-1}\left(s_{n}, t_{0}\right) \\
& \oplus K\left(s, t, s_{n}, t_{n}\right) \\
&\left.\odot u_{m-1}\left(s_{n}, t_{n}\right)\right)
\end{aligned}
$$




$$
\begin{gathered}
\oplus 2\left(\sum_{i=0}^{n-1} K\left(s, t, s_{i}, t_{0}\right)\right. \\
\odot u_{m-1}\left(s_{i}, t_{0}\right) \\
\oplus \sum_{j=0}^{n-1} K\left(s, t, s_{0}, t_{j}\right) \\
\left.\odot u_{m-1}\left(s_{0}, t_{j}\right)\right) \\
\oplus\left(\sum_{i=0}^{n-1} \sum_{j=0}^{n-1} K\left(s, t, s_{i}, t_{j}\right)\right. \\
\left.\left.\odot u_{m-1}\left(s_{i}, t_{j}\right)\right)\right] .
\end{gathered}
$$

The above recursive relation can be written as follows:

$$
\begin{aligned}
& u_{0}(s, t)=f(s, t), \\
& u_{m}(s, t)=f(s, t) \oplus \frac{\lambda h h^{\prime}}{4} \\
& \odot \sum_{i=0}^{n-1} \sum_{j=0}^{n-1}\left(K\left(s, t, s_{i}, t_{j}\right) \odot u_{m-1}\left(s_{i}, t_{j}\right)\right. \\
& \oplus K\left(s, t, s_{i}, t_{j+1}\right) \\
& \odot u_{m-1}\left(s_{i}, t_{j+1}\right) \\
& \oplus K\left(s, t, s_{i+1}, t_{j}\right) \\
& \odot u_{m-1}\left(s_{i+1}, t_{j}\right) \\
& \oplus K\left(s, t, s_{i+1}, t_{j+1}\right) \\
&\left.\odot u_{m-1}\left(s_{i+1}, t_{j+1}\right)\right) .
\end{aligned}
$$

4.1. Error Estimation. Here, we obtain an error estimate between the exact solution and the approximate solution for the given fuzzy Fredholm integral equation (41).

Theorem 26. Consider the 2DFFLIE2 (41) with continuous kernel $K(s, t, x, y)$ having positive sign on $[a, b] \times[c, d] \times[a, b] \times$ $[c, d]$ and suppose that $f$ is continuous on $[a, b] \times[c, d]$. If $B=\lambda M(b-a)(d-c)<1$, where $M=\max _{a \leq s, x \leq b}|K(s, t, x, y)|$, then the iterative procedure (59) converges to the unique solution of (41), F, and the following error estimate holds true:

$$
\begin{gathered}
D^{*}\left(F, u_{m}\right) \leq\left(\frac{B^{m+1}}{1-m}\right) \Gamma_{0}+\left(\frac{B}{4(1-B)}\right) \omega_{[a, b] \times[c, d]}\left(f, h h^{\prime}\right) \\
+\left(\frac{\mu B^{2}+4 \tau B}{4 M(1-B)}\right) \omega_{s t}\left(K, h+h^{\prime}\right),
\end{gathered}
$$

where

$$
\begin{gathered}
\omega_{s t}(K, \delta)=\sup \left\{\left|K\left(s_{1}, t_{1}, x, y\right)-K\left(s_{2}, t_{2}, x, y\right)\right| ;\right. \\
\left.\sqrt{\left(s_{2}-s_{1}\right)^{2}+\left(t_{2}-t_{1}\right)^{2}} \leq \delta\right\} \\
\forall \delta \geq 0, \quad a \leq s_{1}, s_{2} \leq b, \quad c \leq t_{1}, t_{2} \leq d, \\
M_{k}=\sup _{(s, t) \in[a, b] \times[c, d]}\left\|u_{k}(s, t)\right\|_{F}, \\
\Gamma_{k}=\sup _{(s, t) \in[a, b] \times[c, d]}\left\|F_{k}(s, t)\right\|_{F}, \\
\tau=\max _{i=0,1, \ldots, m-1}\left\{M_{i}\right\}, \\
\mu=\max _{i=0,1, \ldots, m-2}\left\{\Gamma_{i}\right\} .
\end{gathered}
$$

Proof. Considering iterative procedure (59), for all $(s, t) \in$ $[a, b] \times[c, d]$, we have

$$
\begin{aligned}
& D\left(F_{1}(s, t), u_{1}(s, t)\right) \\
& =D(f(s, t), f(s, t)) \\
& +D(\lambda \odot(F R) \\
& \cdot \int_{c}^{d}(F R) \int_{a}^{b} K(s, t, x, y) \\
& \odot F_{0}(x, y) d x d y, \\
& \frac{\lambda h h^{\prime}}{4} \odot \sum_{i=0}^{n-1} \sum_{j=0}^{n-1}\left[K\left(s, t, s_{i}, t_{j}\right) \odot F_{0}\left(s_{i}, t_{j}\right)\right. \\
& \oplus K\left(s, t, s_{i}, t_{j+1}\right) \odot F_{0}\left(s_{i}, t_{j+1}\right) \\
& \oplus K\left(s, t, s_{i+1}, t_{j}\right) \odot F_{0}\left(s_{i+1}, t_{j}\right) \\
& \oplus K\left(s, t, s_{i+1}, t_{j+1}\right) \\
& \left.\left.\odot F_{0}\left(s_{i+1}, t_{j+1}\right)\right]\right) \\
& =D\left(\lambda \odot \sum_{i=0}^{n-1} \sum_{j=0}^{n-1}(F R)\right. \\
& \cdot \int_{s_{i}}^{s_{i+1}}(F R) \int_{t_{j}}^{t_{j+1}} K(s, t, x, y) \\
& \odot f(x, y) d x d y,
\end{aligned}
$$




$$
\begin{aligned}
& \frac{\lambda h h^{\prime}}{4} \odot \sum_{i=0}^{n-1} \sum_{j=0}^{n-1}\left[K\left(s, t, s_{i}, t_{j}\right) \odot f\left(s_{i}, t_{j}\right)\right. \\
& \oplus K\left(s, t, s_{i}, t_{j+1}\right) \odot f\left(s_{i}, t_{j+1}\right) \\
& \oplus K\left(s, t, s_{i+1}, t_{j}\right) \odot f\left(s_{i+1}, t_{j}\right) \\
& \oplus K\left(s, t, s_{i+1}, t_{j+1}\right) \\
& \left.\left.\odot f\left(s_{i+1}, t_{j+1}\right)\right]\right) \\
& \leq \lambda \odot \sum_{i=0}^{n-1} \sum_{j=0}^{n-1} D\left((F R) \int_{s_{i}}^{s_{i+1}}(F R) \int_{t_{j}}^{t_{j+1}} K(s, t, x, y)\right. \\
& \odot f(x, y) d x d y \text {, } \\
& \frac{h h^{\prime}}{4} \odot\left[K\left(s, t, s_{i}, t_{j}\right) \odot f\left(s_{i}, t_{j}\right)\right. \\
& \oplus K\left(s, t, s_{i}, t_{j+1}\right) \odot f\left(s_{i}, t_{j+1}\right) \\
& \oplus K\left(s, t, s_{i+1}, t_{j}\right) \odot f\left(s_{i+1}, t_{j}\right) \\
& \oplus K\left(s, t, s_{i+1}, t_{j+1}\right) \\
& \left.\left.\odot f\left(s_{i+1}, t_{j+1}\right)\right]\right) \\
& \leq \lambda \odot \sum_{i=0}^{n-1} \sum_{j=0}^{n-1} D\left((F R) \int_{s_{i}}^{s_{i+1}}(F R) \int_{t_{j}}^{t_{j+1}} K(s, t, x, y)\right. \\
& \odot f(x, y) d x d y \\
& \frac{h h^{\prime}}{4} \odot\left[K(s, t, x, y) \odot f\left(s_{i}, t_{j}\right)\right. \\
& \oplus K(s, t, x, y) \odot f\left(s_{i}, t_{j+1}\right) \\
& \oplus K(s, t, x, y) \odot f\left(s_{i+1}, t_{j}\right) \\
& \oplus K(s, t, x, y) \\
& \left.\left.\odot f\left(s_{i+1}, t_{j+1}\right)\right]\right) \\
& +\lambda \odot \sum_{i=0}^{n-1} \sum_{j=0}^{n-1} D\left(\frac { h h ^ { \prime } } { 4 } \left[K(s, t, x, y) \odot f\left(s_{i}, t_{j}\right)\right.\right. \\
& \oplus K(s, t, x, y) \odot f\left(s_{i}, t_{j+1}\right) \\
& \oplus K(s, t, x, y) \odot f\left(s_{i+1}, t_{j}\right) \\
& \left.\oplus K(s, t, x, y) \odot f\left(s_{i+1}, t_{j+1}\right)\right] \text {, } \\
& \frac{h h^{\prime}}{4} \odot\left[K\left(s, t, s_{i}, t_{j}\right) \odot f\left(s_{i}, t_{j}\right)\right. \\
& \oplus K\left(s, t, s_{i}, t_{j+1}\right) \odot f\left(s_{i}, t_{j+1}\right) \\
& \oplus K\left(s, t, s_{i+1}, t_{j}\right) \odot f\left(s_{i+1}, t_{j}\right)
\end{aligned}
$$

$$
\begin{array}{r}
\oplus K\left(s, t, s_{i+1}, t_{j+1}\right) \\
\left.\left.\odot f\left(s_{i+1}, t_{j+1}\right)\right]\right)
\end{array}
$$$$
\leq \lambda \odot \sum_{i=0}^{n-1} \sum_{j=0}^{n-1}|K(s, t, x, y)|
$$$$
\times D\left((F R) \int_{s_{i}}^{s_{i+1}}(F R) \int_{t_{j}}^{t_{j+1}} f(x, y) d x d y,\right.
$$$$
\frac{h h^{\prime}}{4} \odot\left[f\left(s_{i}, t_{j}\right) \oplus f\left(s_{i}, t_{j+1}\right)\right.
$$$$
\left.\left.\oplus f\left(s_{i+1}, t_{j}\right) \oplus f\left(s_{i+1}, t_{j+1}\right)\right]\right)
$$$$
+\frac{\lambda h h^{\prime}}{4} \odot \sum_{i=0}^{n-1} \sum_{j=0}^{n-1}\left[D \left(K(s, t, x, y) \odot f\left(s_{i}, t_{j}\right),\right.\right.
$$$$
\left.K\left(s, t, s_{i}, t_{j}\right) \odot f\left(s_{i}, t_{j}\right)\right)
$$$$
+D\left(K(s, t, x, y) \odot f\left(s_{i}, t_{j+1}\right),\right.
$$$$
\left.K\left(s, t, s_{i}, t_{j+1}\right) \odot f\left(s_{i}, t_{j+1}\right)\right)
$$$$
+D\left(K(s, t, x, y) \odot f\left(s_{i+1}, t_{j}\right)\right. \text {, }
$$$$
\left.K\left(s, t, s_{i+1}, t_{j}\right) \odot f\left(s_{i+1}, t_{j}\right)\right)
$$$$
+D\left(K(s, t, x, y) \odot f\left(s_{i+1}, t_{j+1}\right)\right. \text {, }
$$$$
\left.\left.K\left(s, t, s_{i+1}, t_{j+1}\right) \odot f\left(s_{i+1}, t_{j+1}\right)\right)\right] .
$$

Using part (ii) of Corollary 19, part (v) of Definition 3, and part (i) of Theorem 13, we obtain

$$
\begin{aligned}
D\left(F_{1}(s, t), u_{1}(s, t)\right) & \\
\leq \frac{\lambda M h h^{\prime}}{4} \sum_{i=0}^{n-1} \sum_{j=0}^{n-1}( & \left.4 \omega_{\left[s_{i}, s_{i+1}\right] \times\left[t_{j}, t_{j+1}\right]}\left(f, \frac{h h^{\prime}}{4}\right)\right) \\
+\frac{\lambda h h^{\prime}}{4} \sum_{i=0}^{n-1} \sum_{j=0}^{n-1}[\mid & K(s, t, x, y)-K\left(s, t, s_{i}, t_{j}\right) \mid \\
& \times D\left(f\left(s_{i}, t_{j}\right), \widetilde{0}\right) \\
& +\left|K(s, t, x, y)-K\left(s, t, s_{i}, t_{j+1}\right)\right| \\
& \times D\left(f\left(s_{i}, t_{j+1}\right), \widetilde{0}\right) \\
& +\left|K(s, t, x, y)-K\left(s, t, s_{i+1}, t_{j}\right)\right| \\
& \times D\left(f\left(s_{i+1}, t_{j}\right), \widetilde{0}\right) \\
& +\left|K(s, t, x, y)-K\left(s, t, s_{i}, t_{j}\right)\right| \\
& \left.\times D\left(f\left(s_{i+1}, t_{j+1}\right), \widetilde{0}\right)\right] .
\end{aligned}
$$


By part (ii) of Theorem 6 and direct computation, it follows that

$$
\begin{aligned}
& D(\left.F_{1}(s, t), u_{1}(s, t)\right) \\
& \leq \frac{\lambda M(b-a)(d-c)}{4} \omega\left(f, h h^{\prime}\right) \\
&+\lambda h h^{\prime} \sum_{i=0}^{n-1} \sum_{j=0}^{n-1}\left(\left|K(s, t, x, y)-K\left(s, t, s_{i}, t_{j}\right)\right|\right) \\
& \quad \sup _{(s, t) \in[a, b] \times[c, d]} D(f(s, t), \widetilde{0}) \\
& \leq \frac{\lambda M(b-a)(d-c)}{4} \omega\left(f, h h^{\prime}\right)+\lambda(b-a)(d-c) \\
& M_{0} \omega_{s t}\left(K, h+h^{\prime}\right)=\frac{B}{4} \omega\left(f, h h^{\prime}\right)+\frac{B}{M} M_{0} \omega_{s t}\left(K, h+h^{\prime}\right) ;
\end{aligned}
$$

therefore, we obtain

$D\left(F_{1}(s, t), u_{1}(s, t)\right) \leq \frac{B}{4} \omega\left(f, h h^{\prime}\right)+\frac{B}{M} M_{0} \omega_{s t}\left(K, h+h^{\prime}\right)$.

Now, since $F_{2}(s, t)=f(s, t)+\lambda \odot$ $(F R) \int_{c}^{d}(F R) \int_{a}^{b} K(s, t, x, y) \odot F_{1}(x, y) d x d y$, we infer that

$$
\begin{aligned}
& D\left(F_{2}(s, t), u_{2}(s, t)\right) \\
& =D(f(s, t), f(s, t)) \\
& +\lambda D\left((F R) \int_{c}^{d}(F R) \int_{a}^{b} K(s, t, x, y) \odot F_{1}(x, y) d x d y,\right. \\
& \quad \frac{h h^{\prime}}{4} \odot \sum_{i=0}^{n-1} \sum_{j=0}^{n-1}\left[K\left(s, t, s_{i}, t_{j}\right) \odot F_{1}\left(s_{i}, t_{j}\right)\right. \\
& \oplus K\left(s, t, s_{i}, t_{j+1}\right) \odot F_{1}\left(s_{i}, t_{j+1}\right) \\
& \oplus K\left(s, t, s_{i+1}, t_{j}\right) \odot F_{1}\left(s_{i+1}, t_{j}\right) \\
& \oplus K\left(s, t, s_{i+1}, t_{j+1}\right) \\
& \left.\left.\odot F_{1}\left(s_{i+1}, t_{j+1}\right)\right]\right) \\
& \leq \lambda \sum_{i=0}^{n-1} \sum_{j=0}^{n-1}\left[D \left((F R) \int_{s_{i}}^{s_{i+1}}(F R) \int_{t_{j}}^{t_{j+1}} K(s, t, x, y)\right.\right.
\end{aligned}
$$$$
\odot F_{1}(x, y) d x d y \text {, }
$$

$$
\begin{aligned}
& \frac{h h^{\prime}}{4} \odot K(s, t, x, y) \\
& \odot\left[F_{1}\left(s_{i}, t_{j}\right) \oplus F_{1}\left(s_{i}, t_{j+1}\right) \oplus F_{1}\left(s_{i+1}, t_{j}\right)\right. \\
& \left.\left.\oplus F_{1}\left(s_{i+1}, t_{j+1}\right)\right]\right) \\
& +\frac{h h^{\prime}}{4} D(K(s, t, x, y) \\
& {\left[F_{1}\left(s_{i}, t_{j}\right) \oplus F_{1}\left(s_{i}, t_{j+1}\right)\right.} \\
& \left.\oplus F_{1}\left(s_{i+1}, t_{j}\right) \oplus F_{1}\left(s_{i+1}, t_{j+1}\right)\right] \\
& K(s, t, x, y) \\
& \times\left[u_{1}\left(s_{i}, t_{j}\right)\right. \\
& \oplus u_{1}\left(s_{i}, t_{j+1}\right) \oplus u_{1}\left(s_{i+1}, t_{j}\right) \\
& \left.\left.\oplus u_{1}\left(s_{i+1}, t_{j+1}\right)\right]\right)
\end{aligned}
$$$$
+\frac{h h^{\prime}}{4} D(K(s, t, x, y)
$$$$
\times\left[u_{1}\left(s_{i}, t_{j}\right)\right.
$$$$
\oplus u_{1}\left(s_{i}, t_{j+1}\right) \oplus u_{1}\left(s_{i+1}, t_{j}\right)
$$$$
\left.\oplus u_{1}\left(s_{i+1}, t_{j+1}\right)\right] \text {, }
$$$$
\left[K\left(s, t, s_{i}, t_{j}\right) \odot u_{1}\left(s_{i}, t_{j}\right)\right.
$$$$
\oplus K\left(s, t, s_{i}, t_{j+1}\right) \odot u_{1}\left(s_{i}, t_{j+1}\right)
$$$$
\oplus K\left(s, t, s_{i+1}, t_{j}\right) \odot u_{1}\left(s_{i+1}, t_{j}\right)
$$$$
\oplus K\left(s, t, s_{i+1}, t_{j+1}\right)
$$$$
\left.\left.\left.\odot u_{1}\left(s_{i+1}, t_{j+1}\right)\right]\right)\right]
$$$$
\leq \frac{\lambda M(b-a)(d-c)}{4} \omega_{[a, b] \times[c, d]}\left(F_{1}, h h^{\prime}\right)
$$$$
+\frac{\lambda M(b-a)(d-c)}{4}\left[D\left(F_{1}\left(s_{i}, t_{j}\right), u_{1}\left(s_{i}, t_{j}\right)\right)\right.
$$$$
+D\left(F_{1}\left(s_{i}, t_{j+1}\right), u_{1}\left(s_{i}, t_{j+1}\right)\right)
$$$$
+D\left(F_{1}\left(s_{i+1}, t_{j}\right), u_{1}\left(s_{i+1}, t_{j}\right)\right)
$$$$
+D\left(F_{1}\left(s_{i+1}, t_{j+1}\right)\right. \text {, }
$$$$
\left.\left.u_{1}\left(s_{i+1}, t_{j+1}\right)\right)\right]
$$$$
+\lambda(b-a)(d-c) M_{1} \omega_{s t}\left(K, h+h^{\prime}\right) ;
$$ 
therefore, we have

$$
\begin{aligned}
D\left(F_{2}\right. & \left.(s, t), u_{2}(s, t)\right) \\
\leq & \frac{B}{4} \omega_{[a, b] \times[c, d]}\left(F_{1}, h h^{\prime}\right) \\
& +\frac{B}{4}\left[D\left(F_{1}\left(s_{i}, t_{j}\right), u_{1}\left(s_{i}, t_{j}\right)\right)\right. \\
& +D\left(F_{1}\left(s_{i}, t_{j+1}\right), u_{1}\left(s_{i}, t_{j+1}\right)\right) \\
& +D\left(F_{1}\left(s_{i+1}, t_{j}\right), u_{1}\left(s_{i+1}, t_{j}\right)\right) \\
& \left.+D\left(F_{1}\left(s_{i+1}, t_{j+1}\right), u_{1}\left(s_{i+1}, t_{j+1}\right)\right)\right] \\
& +\lambda(b-a)(d-c) M_{1} \omega_{s t}\left(K, h+h^{\prime}\right) .
\end{aligned}
$$

By induction for $m \geq 3$, using (45), (46), (59), and (62), we see that

$$
\begin{aligned}
& D\left(F_{m}(s, t), u_{m}(s, t)\right) \\
& \leq \frac{B}{4} \omega_{[a, b] \times[c, d]}\left(F_{1}, h h^{\prime}\right) \\
& +\frac{B}{4}\left[D\left(F_{m-1}\left(s_{i}, t_{j}\right), u_{m-1}\left(s_{i}, t_{j}\right)\right)\right. \\
& \quad+D\left(F_{1}\left(s_{i}, t_{j+1}\right), u_{1}\left(s_{i}, t_{j+1}\right)\right) \\
& \quad+D\left(F_{m-1}\left(s_{i+1}, t_{j}\right), u_{m-1}\left(s_{i+1}, t_{j}\right)\right) \\
& \left.\quad+D\left(F_{m-1}\left(s_{i+1}, t_{j+1}\right), u_{m-1}\left(s_{i+1}, t_{j+1}\right)\right)\right] \\
& +\frac{B}{M} M_{m-1} \omega_{s t}\left(K, h+h^{\prime}\right) ;
\end{aligned}
$$

taking supremum for $(t, s) \in[a, b] \times[c, d]$ from (70), we conclude that

$$
\begin{aligned}
D^{*}( & \left.F_{m}, u_{m}\right) \\
\leq & \frac{B}{4} \omega_{[a, b] \times[c, d]}\left(F_{m-1}, h h^{\prime}\right) \\
& +B D^{*}\left(F_{m-1}, u_{m-1}\right)+\frac{B}{M} M_{m-1} \omega_{s t}\left(K, h+h^{\prime}\right), \\
D^{*}\left(F_{m-1}, u_{m-1}\right) & \\
\leq & \frac{B}{4} \omega_{[a, b] \times[c, d]}\left(F_{m-2}, h h^{\prime}\right) \\
& +B D^{*}\left(F_{m-2}, u_{m-2}\right)+\frac{B}{M} M_{m-2} \omega_{s t}\left(K, h+h^{\prime}\right), \\
D^{*}( & \left.F_{m-2}, u_{m-2}\right) \\
\leq & \frac{B}{4} \omega_{[a, b] \times[c, d]}\left(F_{m-3}, h h^{\prime}\right) \\
& +B D^{*}\left(F_{m-3}, u_{m-3}\right)+\frac{B}{M} M_{m-3} \omega_{s t}\left(K, h+h^{\prime}\right), \\
\vdots & +B D^{*}\left(F_{0}, u_{0}\right)+\frac{B}{M} M_{0} \omega_{s t}\left(K, h+h^{\prime}\right), \\
\leq & \frac{B}{4} \omega_{[a, b] \times[c, d]}\left(F_{0}, h h^{\prime}\right) \\
D^{*}( & \left.F_{1}, u_{1}\right) \\
&
\end{aligned}
$$

and multiplying the above inequalities by $1, B, B^{2}, \ldots, B^{m-1}$, respectively, and summing them, we obtain

$$
\begin{aligned}
& D^{*}\left(F_{m}, u_{m}\right) \\
& \leq \frac{B}{4}\left(\omega_{[a, b] \times[c, d]}\left(F_{m-1}, h h^{\prime}\right)+B \omega_{[a, b] \times[c, d]}\right. \\
& \quad \times\left(F_{m-2}, h h^{\prime}\right) \\
& \left.\quad+\cdots+B^{m-1} \omega_{[a, b] \times[c, d]}\left(f, h h^{\prime}\right)\right) \\
& +\frac{B}{M} \omega_{s t}\left(K, h+h^{\prime}\right)\left(M_{m-1}+B M_{m-2}+B^{2} M_{m-3}\right. \\
& \left.+\cdots+B^{m-1} M_{0}\right) .
\end{aligned}
$$

Since, for $\left(s_{1}, t_{1}\right),\left(s_{2}, t_{2}\right) \in[a, b] \times[c, d]$ with $\left|s_{1}-s_{2}\right| \leq h$, $\left|t_{1}-t_{2}\right| \leq h^{\prime}$, we have

$$
\begin{aligned}
& D\left(F_{m}\left(s_{1}, t_{1}\right), F_{m}\left(s_{2}, t_{2}\right)\right) \\
& =D\left(f\left(s_{1}, t_{1}\right) \oplus \lambda\right. \\
& \otimes \int_{c}^{d} \int_{a}^{b} K\left(s_{1}, t_{1}, x, y\right) \\
& \otimes F_{m-1}(x, y) d x d y \\
& f\left(s_{2}, t_{2}\right) \oplus \lambda \otimes \int_{c}^{d} \int_{a}^{b} K\left(s_{2}, t_{2}, x, y\right) \\
& \left.\otimes F_{m-1}(x, y) d x d y\right) \\
& \leq D\left(f\left(s_{1}, t_{1}\right), f\left(s_{2}, t_{2}\right)\right) \oplus \lambda \\
& \otimes \int_{c}^{d} \int_{a}^{b}\left|K\left(s_{1}, t_{1}, x, y\right)-K\left(s_{2}, t_{2}, x, y\right)\right| \\
& \times D\left(F_{m-1}(x, y), \widetilde{0}\right) d x d y \\
& \leq D\left(f\left(s_{1}, t_{1}\right), f\left(s_{2}, t_{2}\right)\right) \\
& +\frac{B}{M} w_{s t}\left(K, h+h^{\prime}\right) \Gamma_{m-1},
\end{aligned}
$$

we infer that

$$
\begin{aligned}
\omega_{[a, b] \times[c, d]}\left(F_{m}, h h^{\prime}\right) \\
\leq \omega_{[a, b] \times[c, d]}\left(f, h h^{\prime}\right) \\
\quad+\frac{B}{M} \omega_{s t}\left(K, h+h^{\prime}\right) \Gamma_{m-1} .
\end{aligned}
$$


By this inequality and (72), we see that

$$
\begin{aligned}
D^{*}\left(F_{m}, u_{m}\right) & \\
\leq & \frac{B}{4}\left(1+B+B^{2}+\cdots+B^{m-1}\right) \omega_{[a, b] \times[c, d]}\left(f, h h^{\prime}\right) \\
& +\frac{B}{4 M} \omega_{s t}\left(K, h+h^{\prime}\right)\left(B \Gamma_{m-2}+B^{2} \Gamma_{m-3}+\cdots+B^{m-1} \Gamma_{0}\right) \\
& +\frac{B}{M} \omega_{s t}\left(K, h+h^{\prime}\right)\left(M_{m-1}+B M_{m-2}+B^{2} M_{m-3}\right. \\
& \left.+\cdots+B^{m-1} M_{0}\right) \\
= & \frac{B}{4}\left(\frac{1-B^{m}}{1-B}\right) \omega_{[a, b] \times[c, d]}\left(f, h h^{\prime}\right)+\frac{B}{4 M} \omega_{s t}\left(K, h+h^{\prime}\right) \\
\quad & {\left[\left(B \Gamma_{m-2}+B^{2} \Gamma_{m-3}+\cdots+B^{m-1} \Gamma_{0}\right)\right.} \\
& \left.\quad+4\left(M_{m-1}+B M_{m-2}+B^{2} M_{m-3}+\cdots+B^{m-1} M_{0}\right)\right] .
\end{aligned}
$$

By (62) and (63) and since $B<1$, we obtain

$$
\begin{aligned}
D^{*}\left(F_{m}, u_{m}\right) & \\
\leq & \frac{B}{4}\left(\frac{1-B^{m}}{1-B}\right) \omega_{[a, b] \times[c, d]}\left(f, h h^{\prime}\right) \\
& \quad+\frac{B}{4 M} \omega_{s t}\left(K, h+h^{\prime}\right)\left(\frac{B\left(1-B^{m}\right)}{1-B} \mu+\frac{4\left(1-B^{m}\right)}{1-B} \tau\right) \\
\leq & \frac{B}{4(1-B)} \omega_{[a, b] \times[c, d]}\left(f, h h^{\prime}\right) \\
& +\frac{B}{4 M} \omega_{s t}\left(K, h+h^{\prime}\right)\left(\frac{\mu B+4 \tau}{1-B}\right)
\end{aligned}
$$

therefore, we obtain

$$
\begin{aligned}
D^{*}\left(F_{m}, u_{m}\right) \\
\quad \leq\left(\frac{B}{4(1-B)}\right) \omega_{[a, b] \times[c, d]}\left(f, h h^{\prime}\right) \\
\quad+\left(\frac{\mu B^{2}+4 \tau B}{4 M(1-B)}\right) \omega_{s t}\left(K, h+h^{\prime}\right) .
\end{aligned}
$$

By inequalities (77) and (46), we deduce that

$$
\begin{aligned}
D^{*}\left(F, u_{m}\right) \\
\leq D^{*}\left(F, F_{m}\right)+D^{*}\left(F_{m}, u_{m}\right) \\
\leq\left(\frac{B^{m+1}}{1-B}\right) \Gamma_{0}+\left(\frac{B}{4(1-B)}\right) \omega_{[a, b] \times[c, d]}\left(f, h h^{\prime}\right) \\
\quad+\left(\frac{\mu B^{2}+4 \tau B}{4 M(1-B)}\right) \omega_{s t}\left(K, h+h^{\prime}\right) .
\end{aligned}
$$

Remark 27 . Since $B<1$, it is easy to see that

$$
\lim _{\substack{m \rightarrow \infty \\ h, h^{\prime} \rightarrow 0}} D^{*}\left(F, u_{m}\right)=0
$$

which shows the convergence of the method.

\section{Numerical Experiments}

The proposed iterative method of successive approximations was tested on three numerical examples to provide the accuracy and the convergence of the method and illustrate the correctness of the theoretical results. In these examples, we assumed that $[a, b] \times[c, d]=[0,1] \times[0,1], \lambda=1$, and we performed the algorithm in point $\left[s_{0}, t_{0}\right]=[0.5,0.5]$.

Example 1. Assume that

$$
F(s, t)=f(s, t) \oplus \iint_{0}^{1} K(s, t, x, y) \odot F(x, y) d x d y
$$

where

$$
\begin{aligned}
f(s, t, r) & =(\underline{f}(s, t, r), \bar{f}(s, t, r)), \\
\underline{f}(s, t, r) & =\left(r^{2}+r\right) s \sin \frac{t}{2}, \\
\bar{f}(s, t, r) & =\left(4-r^{3}-r\right) s \sin \frac{t}{2}, \\
K(s, t, x, y) & =s^{2} t x ;
\end{aligned}
$$

the exact solution is given by

$$
\begin{aligned}
& F(s, t, r)=(\underline{F}(s, t, r), \bar{F}(s, t, r)), \\
& \underline{F}(s, t, r)=\left(r^{2}+r\right)\left(s \sin \frac{t}{2}-\frac{16}{21}\left(\cos \frac{1}{2}-1\right) s^{2} t\right), \\
& \bar{F}(s, t, r)=\left(4-r^{3}-r\right)\left(s \sin \frac{t}{2}-\frac{16}{21}\left(\cos \frac{1}{2}-1\right) s^{2} t\right) .
\end{aligned}
$$

To obtain numerical solution, we apply the proposed method. To compare numerical and exact solutions, see Table 1.

Example 2. Consider (80) with

$$
\begin{aligned}
f(s, t, r) & =(\underline{f}(s, t, r), \bar{f}(s, t, r)), \\
\underline{f}(s, t, r) & =r\left(\frac{1}{3} r+\frac{8}{3}\right)\left(1+s+t-\frac{7}{12} s t\right), \\
\bar{f}(s, t, r) & =\left(2 r^{2}-4 r+5\right)\left(1+s+t-\frac{7}{12} s t\right), \\
K(s, t, x, y) & =s t x y
\end{aligned}
$$


TABLE 1: Numerical results on the level sets for Example 1 in $\left(s_{0}, t_{0}\right)=(0.5,0.5)$.

\begin{tabular}{lcccccccc}
\hline \multirow{2}{*}{-level } & \multicolumn{2}{c}{$m=5, n=10$} & \multicolumn{2}{c}{$m=5, n=20$} & \multicolumn{2}{c}{$m=7, n=10$} & \multicolumn{2}{c}{$m=7, n=20$} \\
& $\left|\underline{F}-\underline{u}_{m}\right|$ & $\left|\bar{F}-\bar{u}_{m}\right|$ & $\left|\underline{F}-\underline{u}_{m}\right|$ & $\left|\bar{F}-\bar{u}_{m}\right|$ & $\left|\underline{F}-\underline{u}_{m}\right|$ & $\left|\bar{F}-\bar{u}_{m}\right|$ & $\left|\underline{F}-\underline{u}_{m}\right|$ & $\left|\bar{F}-\bar{u}_{m}\right|$ \\
\hline 0.00 & 0.000000 & 0.000657 & 0.000000 & 0.000661 & 0.000000 & 0.000008 & 0.000000 & 0.000003 \\
0.25 & 0.000067 & 0.000427 & 0.000051 & 0.000617 & 0.000011 & 0.000053 & 0.000001 & 0.000005 \\
0.50 & 0.000086 & 0.000586 & 0.000024 & 0.000258 & 0.000066 & 0.000815 & 0.000043 & 0.000001 \\
0.75 & 0.000150 & 0.000423 & 0.000022 & 0.000367 & 0.000069 & 0.000499 & 0.000008 & 0.000006 \\
1.00 & 0.000229 & 0.000329 & 0.000131 & 0.000221 & 0.000154 & 0.000154 & 0.000005 & 0.000005 \\
\hline
\end{tabular}

TABle 2: Numerical results on the level sets for Example 2 in $\left(s_{0}, t_{0}\right)=(0.5,0.5)$.

\begin{tabular}{lcccc}
\hline \multirow{2}{*}{$r$-level } & \multicolumn{2}{c}{$m=4, n=10$} & \multicolumn{2}{c}{$m=8, n=20$} \\
& $\left|\underline{F}-\underline{u}_{m}\right|$ & $\left|\bar{F}-\bar{u}_{m}\right|$ & $\left|\underline{F}-\underline{u}_{m}\right|$ & $\left|\bar{F}-\bar{u}_{m}\right|$ \\
\hline 0.0 & 0.000000 & 0.000000 & 0.000000 & 0.000000 \\
0.2 & 0.000003 & 0.000008 & 0.000000 & 0.000002 \\
0.4 & 0.000007 & 0.000011 & 0.000000 & 0.000008 \\
0.6 & 0.000004 & 0.000006 & 0.000001 & 0.000000 \\
0.8 & 0.000000 & 0.000005 & 0.000000 & 0.000000 \\
1.0 & 0.000000 & 0.000000 & 0.000000 & 0.000000 \\
\hline
\end{tabular}

and exact solution

$$
\begin{aligned}
F(s, t, r)= & (\underline{F}(s, t, r), \bar{F}(s, t, r)), \\
= & \left(r\left(\frac{1}{3} r+\frac{8}{3}\right)(s+t+1),\right. \\
& \left.\left(2 r^{2}-4 r+5\right)(s+t+1)\right) .
\end{aligned}
$$

We perform the proposed method and obtain numerical solution. Comparison of these two results is presented in Table 2.

Example 3. The integral equation (80) with

$$
\begin{aligned}
f(s, t, r) & =(\underline{f}(s, t, r), \bar{f}(s, t, r)), \\
\underline{f}(s, t, r) & =(2 r \cos (1-r)-1)\left(1+s^{2}+t-\frac{13}{24}(s+t)\right), \\
\bar{f}(s, t, r) & =\left(2-\sin \frac{r \pi}{2}\right)\left(1+s^{2}+t-\frac{13}{24}(s+t)\right), \\
K(s, t, x, y) & =(s+t) x y
\end{aligned}
$$

has the exact solution

$$
\begin{aligned}
F(s, t, r)= & (\underline{F}(s, t, r), \bar{F}(s, t, r)), \\
= & \left((2 r \cos (1-r)-1)\left(s^{2}+t+1\right),\right. \\
& \left.\left(2-\sin \frac{r \pi}{2}\right)\left(s^{2}+t+1\right)\right) .
\end{aligned}
$$

For this linear example, we apply our proposed iterative method and obtain numerical results that can be viewed in Table 3.
TABle 3: Numerical results on the level sets for Example 3 in $\left(s_{0}, t_{0}\right)=(0.5,0.5)$.

\begin{tabular}{lcccc}
\hline \multirow{2}{*}{-level } & \multicolumn{2}{c}{$m=5, n=20$} & \multicolumn{2}{c}{$m=5, n=40$} \\
& $\left|\underline{F}-\underline{u}_{m}\right|$ & $\left|\bar{F}-\bar{u}_{m}\right|$ & $\left|\underline{F}-\underline{u}_{m}\right|$ & $\left|\bar{F}-\bar{u}_{m}\right|$ \\
\hline 0.0 & 0.000047 & 0.000028 & 0.000008 & 0.000003 \\
0.2 & 0.000039 & 0.000086 & 0.000006 & 0.000009 \\
0.4 & 0.000009 & 0.000008 & 0.000003 & 0.000000 \\
0.6 & 0.000004 & 0.000005 & 0.000001 & 0.000000 \\
0.8 & 0.000012 & 0.000033 & 0.000007 & 0.000002 \\
1.0 & 0.000006 & 0.000004 & 0.000000 & 0.000000 \\
\hline
\end{tabular}

\section{Conclusions}

In this paper, we introduced 2D fuzzy mappings and defined 2D fuzzy integrals. Quadrature rules to approximate the solution of 2D fuzzy integrals are given. We established the theorem of existence of unique solution of 2DFFLIE2, and we have proved it by using Banach's fixed point principle. Moreover, to approximate the solution of 2DFFLIE2, we have proposed an iterative algorithm based on method of successive approximations. The convergence to the unique solution in our iterative method is investigated. The presented numerical experiments show that the method applies well for 2DFFLIE2.

\section{Conflict of Interests}

The authors declare that there is no conflict of interests regarding the publication of this paper.

\section{Acknowledgments}

The authors would like to thank the editor and anonymous referees for various suggestions which have led to an improvement in both the quality and clarity of the paper.

\section{References}

[1] H.-J. Zimmermann, Fuzzy Set Theory and Its Applications, Kluwer Academic, New York, NY, USA, 1996.

[2] C. Wu and Z. Gong, "On Henstock integral of fuzzy-numbervalued functions-I," Fuzzy Sets and Systems, vol. 120, no. 3, pp. 523-532, 2001.

[3] H.-C. Wu, "The fuzzy Riemann integral and its numerical integration," Fuzzy Sets and Systems, vol. 110, no. 1, pp. 1-25, 2000 . 
[4] M. Friedman, M. Ma, and A. Kandel, "Solutions to fuzzy integral equations with arbitrary kernels," International Journal of Approximate Reasoning, vol. 20, no. 3, pp. 249-262, 1999.

[5] H.-C. Wu, "Evaluate fuzzy Riemann integrals using the Monte Carlo method," Journal of Mathematical Analysis and Applications, vol. 264, no. 2, pp. 324-343, 2001.

[6] B. Bede and S. G. Gal, "Quadrature rules for integrals of fuzzynumber-valued functions," Fuzzy Sets and Systems, vol. 145, no. 3, pp. 359-380, 2004.

[7] T. Allahviranloo and M. Otadi, "Gaussian quadratures for approximate of fuzzy integrals," Applied Mathematics and Computation, vol. 170, no. 2, pp. 874-885, 2005.

[8] X. Wu, "Optimal fuzzy quadrature formula for classes of fuzzynumber-valued functions of Lipschitz type," Fuzzy Sets and Systems, vol. 223, pp. 100-108, 2013.

[9] T. Allahviranloo, S. Abbasbandy, S. Salahshour, and A. Hakimzadeh, "A new method for solving fuzzy linear differential equations," Computing, vol. 92, no. 2, pp. 181-197, 2011.

[10] S. Salahshour and T. Allahviranloo, "Application of fuzzy differential transform method for solving fuzzy Volterra integral equations," Applied Mathematical Modelling, vol. 37, no. 3, pp. 1016-1027, 2013.

[11] T. Allahviranloo, M. Khezerloo, O. Sedaghatfar, and S. Salahshour, "Toward the existence and uniqueness of solutions of second-order fuzzy Volterra integro-differential equations with fuzzy kernel," Neural Computing and Applications, vol. 22, no. 1, supplement, pp. 133-141, 2013.

[12] R. Ezzati and S. Ziari, "Numerical solution of two-dimensional fuzzy Fredholm integral equations of the second kind using fuzzy bivariate Bernstein polynomials," International Journal of Fuzzy Systems, vol. 15, no. 1, pp. 84-89, 2013.

[13] R. Goetschel Jr. and W. Voxman, "Elementary fuzzy calculus," Fuzzy Sets and Systems, vol. 18, no. 1, pp. 31-43, 1986.

[14] G. A. Anastassiou and S. G. Gal, "On a fuzzy trigonometric approximation theorem of Weierstrass-type," Journal of Fuzzy Mathematics, vol. 9, no. 3, pp. 701-708, 2001.

[15] M. Friedman, M. Ma, and A. Kandel, "Numerical solutions of fuzzy differential and integral equations," Fuzzy Sets and Systems, vol. 106, no. 1, pp. 35-48, 1999.

[16] G. A. Anastassiou, Fuzzy Mathematics: Approximation Theory, Springer, Berlin, Germany, 2010.

[17] S. G. Gal, "Approximation theory in fuzzy setting," in Handbook of Analytic Computational Methods in Applied Mathematics, G. A. Anastassiou, Ed., chapter 13, pp. 617-666, Chapman \& Hall/CRC, New York, NY, USA, 2000.

[18] Y. Feng, "Fuzzy-valued mappings with finite variation, fuzzyvalued measures and fuzzy-valued Lebesgue-Stieltjes integrals," Fuzzy Sets and Systems, vol. 121, no. 2, pp. 227-236, 2001. 


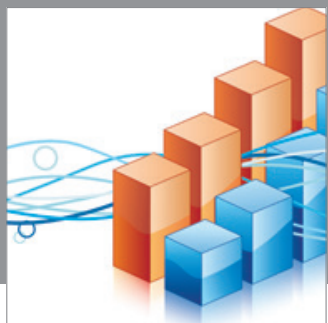

Advances in

Operations Research

mansans

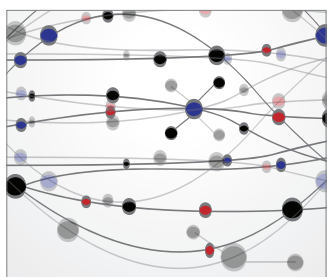

The Scientific World Journal
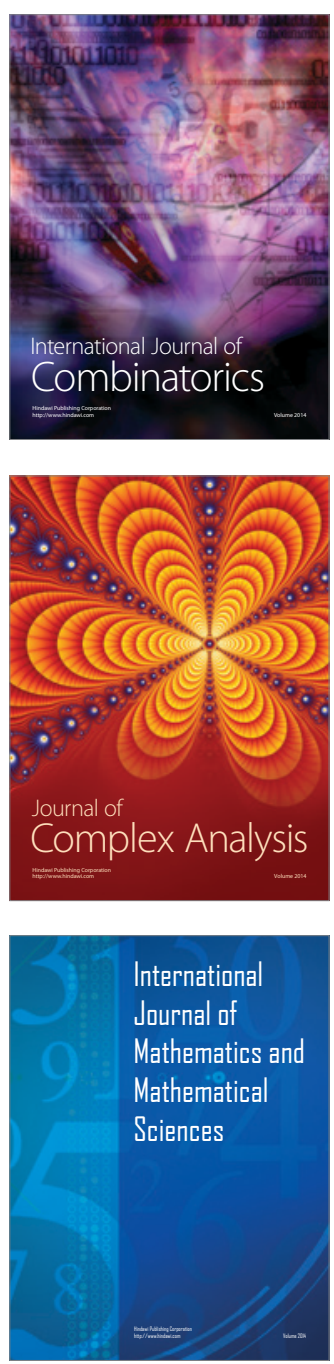
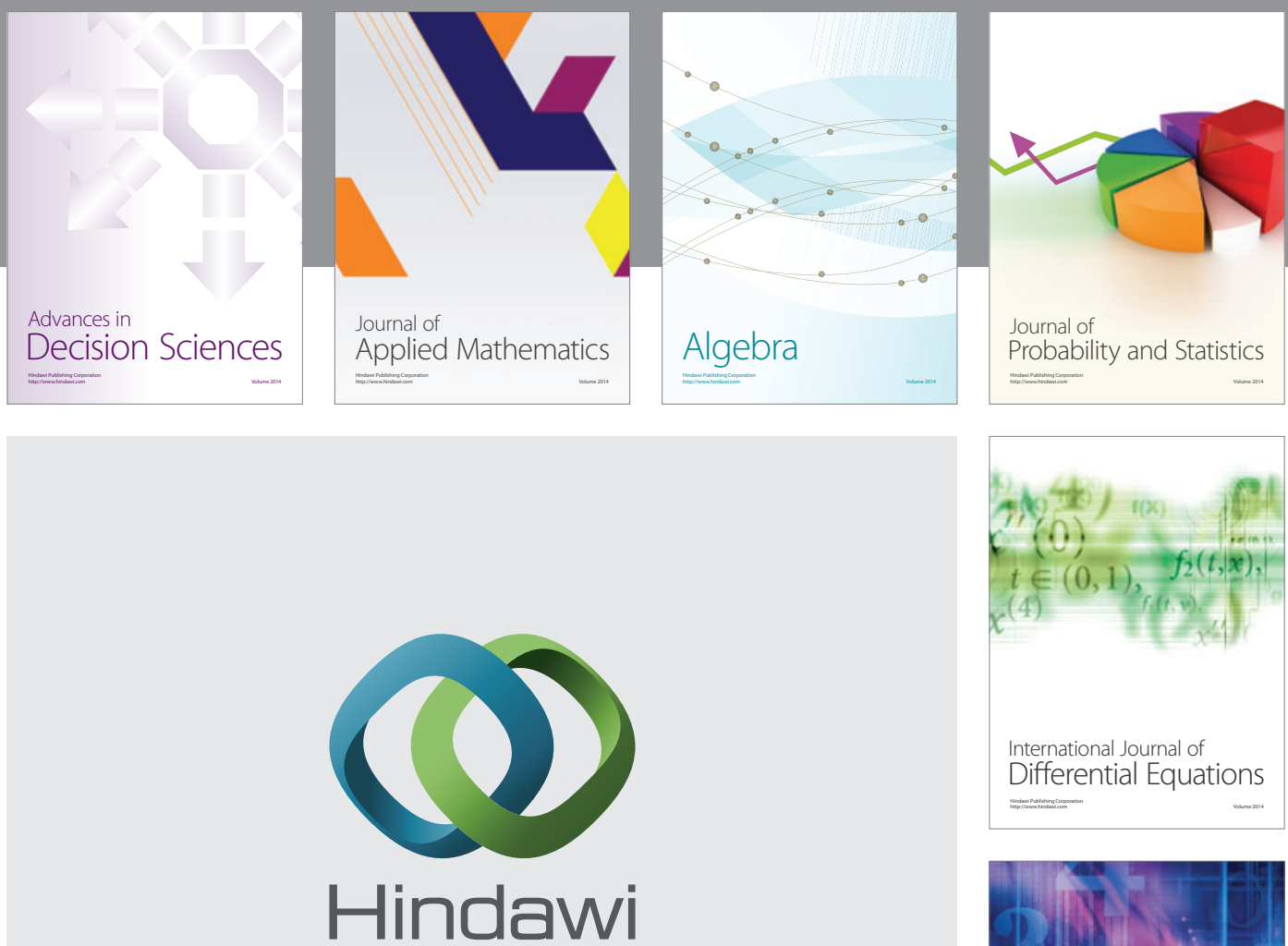

Submit your manuscripts at http://www.hindawi.com
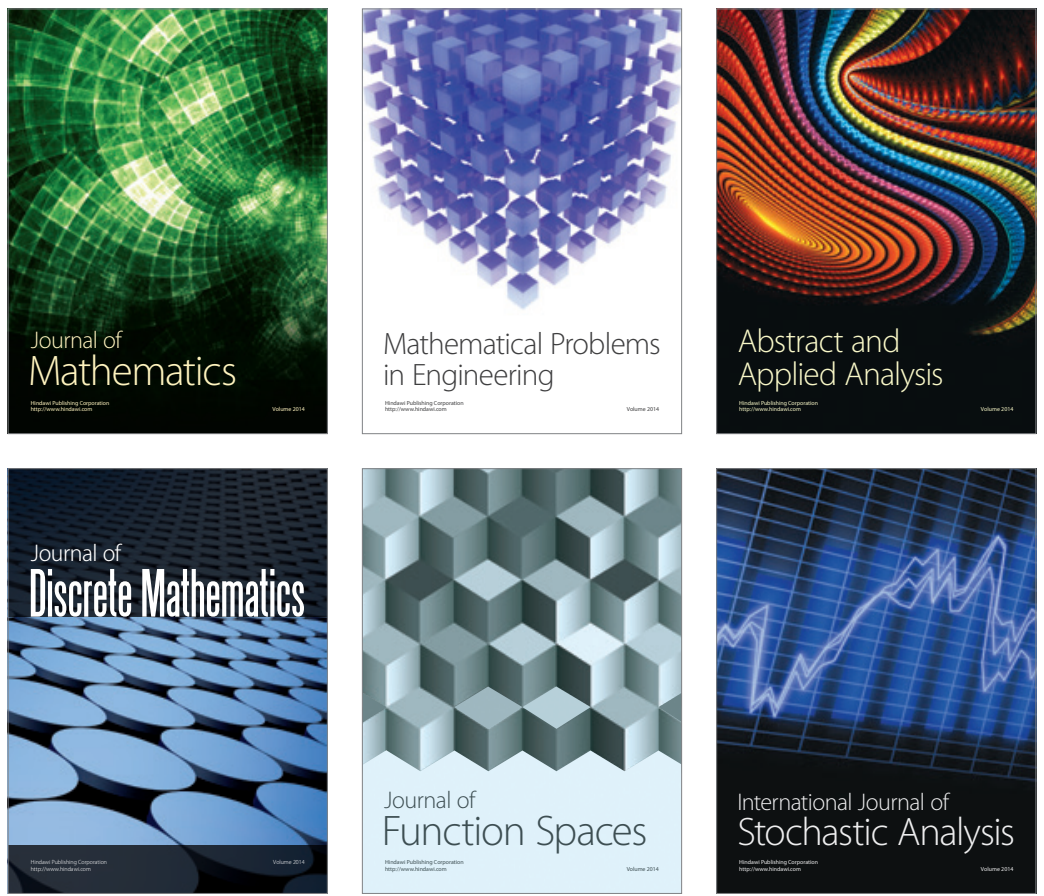

Journal of

Function Spaces

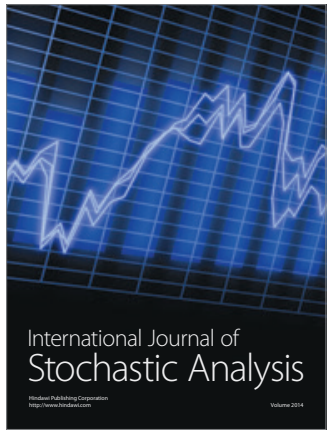

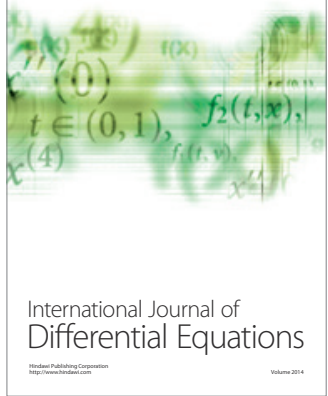
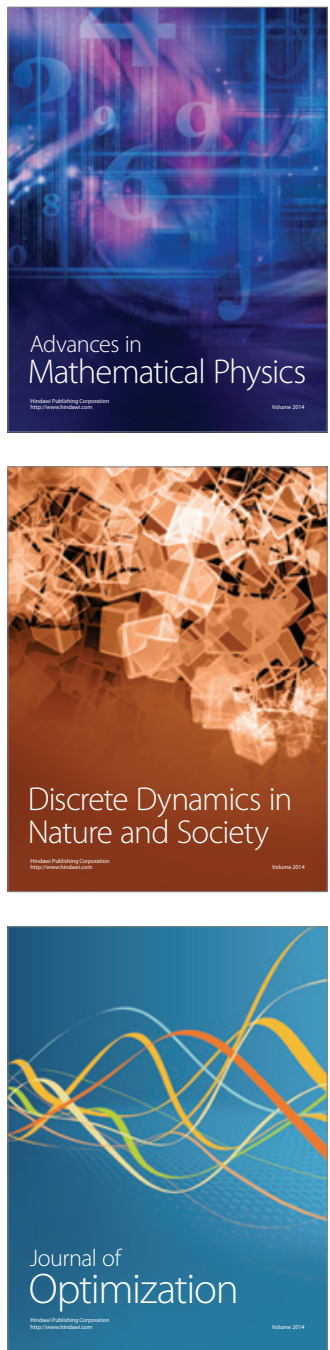